\title{
The Chemical Properties of Milky Way and M31 Globular Clusters: I. A Comparative Study
}

\author{
Michael A. Beasley \\ Lick Observatory, University of California, Santa Cruz, CA 95064, USA \\ mbeasley@ucolick.org \\ Jean P. Brodie \\ Lick Observatory, University of California, Santa Cruz, CA 95064, USA \\ brodie@ucolick.org \\ Jay Strader \\ Lick Observatory, University of California, Santa Cruz, CA 95064, USA \\ strader@ucolick.org \\ Duncan A. Forbes \\ Centre for Astrophysics 85 Supercomputing, Swinburne University, Hawthorn, VIC 3122, Australia \\ dforbes@astro.swin.edu.au \\ Robert N. Proctor \\ Centre for Astrophysics 8 Supercomputing, Swinburne University, Hawthorn, VIC 3122, Australia \\ rproctor@astro.swin.edu.au \\ Pauline Barmby \\ Harvard-Smithsonian Center for Astrophysics, 60 Garden Street, Cambridge, MA 02138, USA \\ pbarmby@cfa.harvard.edu \\ John P. Huchra \\ Harvard-Smithsonian Center for Astrophysics, 60 Garden Street, Cambridge, MA 02138, USA \\ huchra@cfa.harvard.edu
}

ABSTRACT 
A comparative analysis is performed between high-quality integrated spectral indices of 30 globular clusters in M31, 20 Milky Way globular clusters, and a sample of field and cluster elliptical galaxies. We find that the Lick CN indices in the M31 and Galactic clusters are enhanced relative to the bulges of the Milky Way, M31, and elliptical spheroids, in agreement with Burstein et al. Although not particularly evident in the Lick CN indices, the near-UV cyanogen feature $(\lambda 3883)$ is strongly enhanced with respect to the Galactic globulars at metallicities, $-1.5<[\mathrm{Fe} / \mathrm{H}]<-0.3$. Carbon shows signs of varying amongst these two groups. For $[\mathrm{Fe} / \mathrm{H}]>-0.8$, we observe no systematic differences in the $\mathrm{H} \delta, \mathrm{H} \gamma$, or $\mathrm{H} \beta$ indices between the M31 and Galactic globulars, in contrast to previous studies. The elliptical galaxy sample lies offset from the loci of the globular clusters in the both the Cyanogen-[MgFe], and Balmer-line- $[\mathrm{MgFe}]$ planes. Six of the M31 clusters appear young, and are projected onto the M31 disk. Population synthesis models suggest that these are metal-rich clusters with ages 100-800 Myr, metallicities $-0.20 \leq[\mathrm{Fe} / \mathrm{H}] \leq 0.35$, and masses $0.7 \sim 7.0 \times 10^{4} \mathrm{M}_{\odot}$. Two other young clusters are Hubble V in NGC 205, observed as a template, and an older ( $\sim 3$ Gyr) cluster some $7 \mathrm{kpc}$ away from the plane of the disk. The six clusters projected onto the disk show signs of rotation similar to the HI gas in M31, and three clusters exhibit thin disk kinematics (Morrison et al.). Dynamical mass estimates and detailed structural parameters are required for these objects to determine whether they are massive open clusters or globular clusters. If they are the latter, our findings suggest globular clusters may trace the build up of galaxy disks. In either case, we conclude that these clusters are part of a young, metal-rich disk cluster system in M31, possibly as young as 1 Gyr old.

Subject headings: globular clusters: general - galaxies: individual: M31 - galaxies: individual

\section{Introduction}

Understanding the formation and evolution of disk galaxies is a crucial aspect of galaxy formation studies (Freeman \& Bland-Hawthorn 2002). Much of our knowledge about such disks is based upon detailed observations of the Milky Way, and in this regard, its globular clusters (GCs) have played a prominent role. The Milky Way possesses a retinue of $\sim 150$ GCs which, while a minor contributer to the total mass budget of the Galaxy (they constitute some $\sim 3 \times 10^{7} \mathrm{M}_{\odot}$ in total, or $\sim 0.1 \%$ of the baryonic mass of the Milky Way), have proved invaluable for understanding its structure, stellar populations, and mass aggregation history (e.g., Searle \& Zinn 1978). They comprise of at least two components, a spatially extended, metal-poor, pressure-supported system, and a metal-rich, centrally concentrated system which exhibits some rotation (Zinn 1985). Although initially thought to be associated with the thick disk (Zinn 1985), the majority of these metal-rich clusters are now believed to be associated with the bulge (Frenk \& White 1982; Minitti 1995; Coté 
1999; Forbes, Brodie \& Larsen 2001).

To date, all the GCs in the Galaxy for which detailed color-magnitude diagrams have been obtained appear 'old' (i.e., >8 Gyr), although a subset of metal-rich GCs may be up to $\sim 20 \%$ younger than the inner-halo GCs (Rosenberg et al. 1999; Salaris \& Weiss 2002). There is also evidence for a spread of ages among the outer-halo GCs, suggesting either a clumpy collapse (Searle \& Zinn 1978) or accreted origin (e.g., Coté et al. 2000; Bellazzini et al. 2003). The significance of such accretions are currently the subject of much debate in light of the presently favored hierarchical clustering paradigm (Brook et al. 2003; Martin et al. 2004; Forbes, Strader \& Brodie 2004).

Notwithstanding our increasingly detailed knowledge regarding the Milky Way GC system, surprisingly little is known about the GC systems of spiral galaxies in general. This is largely a result of the difficulties involved in observing what are relatively poor GC systems (compared with luminous ellipticals), that are often projected against an inhomogeneous background. However, the gaps in our knowledge about the GC systems of external spirals is slowly changing, with increasingly detailed photometric (e.g., Goudfrooij et al. 2003; Rhode 2003; Chandar, Whitmore \& Lee 2004) and spectroscopic studies (Chandar, Bianchi \& Ford 1999; Schroder et al. 2002; Olsen et al. 2004).

In this regard, our close companion, the Andromeda spiral (M31; D 780 kpc, Holland 1998; Stanek \& Garnavich 1998) provides an important test-bed for the generality of any Milky Way formation theories. It is of similar Hubble type ( $\mathrm{Sb}$ ) to the Milky Way, although it is possibly less massive (Coté et al. 2000; Gottesman, Hunter \& Boonysait 2002). Unlike the Milky Way, M31 appears to have a predominantly metal-rich stellar halo (e.g., Holland, Fahlman \& Richer 1996; Bellazini et al. 2003; Rich et al. 2004).

M31 possesses the most populous GC system in the Local Group. van den Bergh (1999) estimated a total GC system of $400 \pm 55$, which is at least a factor of two larger than that of the Milky Way. Previous studies to catalog the M31 GC system have included Sargent et al. (1977), Battistini et al. (1980, 1987, 1993) and Crampton et al. (1985). Recently, Barmby et al. (2000) published a comprehensive photometric and spectroscopic catalog of 435 GCs and GC candidates. These authors showed that the M31 GC system could be divided into metal-poor and metal-rich sub-populations, with their kinematics separating into halo and bulge/disk components similar to that of the Milky Way. Perrett et al. (2002) obtained intermediate resolution spectra for a subset of $\sim 200$ M31 clusters, offering a great improvement in velocity accuracy over previous efforts. This increase in accuracy lead a recent study by Morrison et al. (2004) to kinematically identify what is possibly a thin disk population of M31 GCs. Such a system has not been observed in the Milky Way.

van den Bergh (1969) was the first to study the metallicity distributions of GCs in M31, from integrated spectra and $U B V$ photometry obtained with the Hale 200-inch. He concluded that on average, the M31 GC system was more metal-rich than both the Milky Way and Fornax dwarf GC systems. In an important contribution, Burstein et al. (1984) comprehensively discussed differences in the stellar content of GCs in the Milky Way and M31. From integrated spectra, they found that at 
high metallicity, at a given metal-line strength the hydrogen lines (specifically H $\beta$ ) of the M31 GCs were systematically stronger than their Galactic counterparts. They explored a number of possible explanations for this phenomenon, and concluded that the most likely explanation was for an age difference in the GCs, in that the M31 GCs were systematically younger than the Milky Way's. They also found that CN in the intermediate- to metal-rich M31 GCs was significantly enhanced (by several tenths of a magnitude) when compared to elliptical galaxies. This CN enhancement could not be demonstrated at low metallicities. No satisfactory explanation which could explain both the 'CN' anomaly and the enhanced Balmer indices has been put forward.

In a series of papers Brodie \& Huchra 1990, 1991; Huchra, Brodie \& Kent 1991) investigated the metallicity and abundances of M31 GCs using a more extensive sample of integrated spectra. In general, they found that the properties of the M31 GC system were similar to those of the Galactic system. Notable exceptions were that the $\mathrm{CN}$ and $\mathrm{H}+\mathrm{K}$ line-strengths were higher in the M31 GCs (at a given metallicity) than the Galactic GCs, supporting the Burstein et al. (1984) findings. However, with the exception of a few individual cases, they saw no systematic evidence for an enhancement in $\mathrm{H} \beta$ in M31 GCs.

The CN enhancement in the M31 GCs is now reasonably well established (Trippico 1989; Ponder et al. 1998; Li \& Burstein 2004), whilst the differences in $\mathrm{H} \beta$ are somewhat more controversial (see Trippico 1994 for a short review). However, it is important to note that in the above studies, the most metal-rich Galactic GCs were not well represented, mostly due to the difficulty of observing these objects against the bright background presented by the Galaxy. If metal-rich GCs are associated with spiral bulges (Frenk \& White 1982; Coté 1999; Forbes et al. 2001), then M31's bulge, which is an order-of-magnitude more massive than that of the Milky Way should host many more metal-rich bulge GCs, resulting in an unequal comparison between the two GC populations (see Trager 2004).

In this paper, we compare Galactic GC data, which include a number of 'bulge GCs' (Cohen, Blakeslee, \& Rhyzhov 1998; Puzia et al. 2002) and newly analyzed spectroscopic data for M31 GCs in order to investigate some of these issues in detail. In a forthcoming paper (Beasley et al., in preparation (Paper II)), we compare these full datasets to contemporary stellar population models in order to derive parameters such as metallicity, age and $\alpha$-to-iron abundance ratios.

The outline of this paper is as follows: In Section 2 we discuss the samples under study and new data reduction steps required. Next, details of the spectroscopic system are given in Section 3 . In Section 4 we look at the behavior of the Balmer and CN indices of the GCs, compared to recent elliptical galaxy data. In Section 5 we look in detail at eight young clusters in the M31 spectroscopic sample. Finally, we present our discussion and conclusions in Section 6. 


\section{Globular Cluster Data}

\subsection{M31 Globular Clusters}

The integrated spectra of M31 globular clusters used in this study were previously described in Barmby et al. (2000). These authors obtained radial velocities for the clusters, and derived metallicities using the empirical calibrations of Brodie \& Huchra (1990). We have not used the MMT spectra described in Barmby et al. (2000), since these spectra are generally of insufficient signal-to-noise $(\mathrm{S} / \mathrm{N})$ for accurate line-strength analysis. In the following, we describe this dataset, and outline the data reduction steps which differ from Barmby et al. (2000).

Integrated spectra for $42 \mathrm{GC}$ candidates in M31 were obtained with the Low Resolution Imaging Spectrograph (LRIS; Oke et al. 1995) on the Keck-I telescope. The observations were undertaken in 1995, September 21st-23rd, and in 1996, December 8th, with some effort to obtain spectra of clusters in the central galactic regions of M31. Candidates were drawn from the catalog of Barmby et al. (2000), with the majority originating from the work of Battistini et al. (1987). Integrations were typically 5 minutes per target, which with characteristic magnitudes of $\mathrm{V} \sim 17$, yielded spectra with S/N of $20-200$ per resolution element. Typical seeing was 1 arcsec during these observations, and the same 1 arcsec $\times 1.5$ arcmin longslit was used for all clusters. A 600 line $\mathrm{mm}^{-1}$ grating was used yielding a $1.2 \AA$ pixel $^{-1}$ dispersion, and a full-width, half-maximum resolution (FWHM) of $5 \AA$. This resolution was determined from measuring a series of isolated arc-lines in the comparison lamp spectra, and found to be constant over our spectral range 3670-6200.

The data reduction steps of bias subtraction, flat-fielding and sky-subtraction were performed using standard packages in $\mathrm{IRAF}^{1}$. The spectra were wavelength calibrated using arc lamp spectra, covering the full spectral range, which were interspersed amongst the M31 observations.

The accuracy of the relative flux calibrations of the spectra can have a significant impact on the measurement of certain broad line-strength indices. For these data, this is true of the $\operatorname{Lick} \mathrm{CN}_{1}$, $\mathrm{CN}_{2}, \mathrm{Mg}_{1}, \mathrm{Mg}_{2}$ and $\mathrm{TiO}_{1}$ indices. For the present study, the original un-flux calibrated spectra from Barmby et al. have been re-fluxed using a larger series of flux standards taken during the time of the LRIS observations. These flux standards, BD $+28^{\circ} 4211, \mathrm{BD}+25^{\circ} 4655$, Feige 110 and GD 50 were all selected from the faint spectrophotometric standard star list of Oke (1990). The standards were all taken with the same slit size $(1 \operatorname{arcsec} \times 1.5 \operatorname{arcsec})$ as the program observations, and covered a range of airmasses. In general, the new flux calibrations agree to within $\sim 10 \%$ of the old calibrations. However, we do observe significant differences in the spectra which exhibit earlier spectral-types (see Section 5), which we attribute to differences in airmass between the original flux standards and the program clusters. The following analysis is based on the newly flux-calibrated M31 data.

\footnotetext{
${ }^{1}$ IRAF is distributed by the National Optical Astronomy Observatories, which are operated by the Association of Universities for Research in Astronomy, Inc., under co-operative agreement with the National Science Foundation
} 
Reliable line-strength measurements require a $\mathrm{S} / \mathrm{N}$ such that the index and pseudo-continuum are well defined. Therefore, we have focused on a high $\mathrm{S} / \mathrm{N}\left(>30\right.$ pixel $\left.^{-1}\right)$ subset of these data. From 42 spectra of candidate M31 GCs, 36 are of sufficient S/N for a detailed analysis of their line-strength indices. Five clusters, all drawn from the near-nucleus cluster catalog of Battistini et al. (1993), we have identified as being likely foreground G-dwarfs of the basis of their spectra. These objects exhibit weak $\mathrm{CN}$ and $\mathrm{TiO}$ molecular bands, closely following the dwarf sequences of the Lick stellar library, rather than that of the majority of the M31 GCs and Lick giants. Moreover, a measure of their surface gravities based upon the SrII/FeI diagnostic (Rose 1985) places them clearly on the locus of Lick dwarfs, rather than that of a dwarf-giant mix expected for GCs (Tripicco 1989) ${ }^{2}$. These objects (NB 68, NB 74, NB 81, NB 87 and NB 91) were removed from the sample. The basic data for all the M31 clusters which satisfy our S/N and 'star cluster' criteria are listed in Table 1. The spatial distribution of the sample with respect to M31 is shown in Figure 1.

To anticipate some of the results of this study, spectra for eight of the M31 GC candidates have spectral characteristics typical of young stellar populations or A-F stars; weak metal-lines, relatively strong absorption in the hydrogen Balmer lines and relatively blue continua when compared to 'classical' G or K-type GC spectra. These are discussed separately in Section 5 and not included in the main analysis.

\subsection{Galactic Globular Clusters}

Galactic GCs play a vital role in the interpretation of the integrated light of more distant GC systems. They not only allow for direct comparative studies, but also provide important benchmarks for stellar population models since they generally have independently derived ages, metallicities and often, heavy-element abundance ratios. Increasingly, high-quality integrated spectra for Galactic GCs are appearing in the literature, and we make use of two such studies.

Cohen et al. (1998; henceforth CBR98) obtained LRIS long-slit spectra for 12 Galactic GCs in order to compare with their multi-slit M87 GC data. These spectra were obtained by scanning $\sim 90$ arcsec across the cores of the Galactic GCs in order to synthesize a larger aperture, and thus obtain a representative integrated spectrum. These data are all of high $\mathrm{S} / \mathrm{N}$, but were taken using differing grating angles yielding 6 spectra with wavelength ranges in the interval $3800-6500 \AA$, and 6 spectra slightly shifted to the red, with wavelength ranges of $4500-7200 \AA$. The LRIS 600 lines $\mathrm{mm}^{-1}$ grating used yielded a dispersion of $1.2 \AA$ pixel $^{-1}$, and resolution (FWHM) of $\sim 6 \AA$.

CBR98 did not flux-calibrate these data, and measured indices by individually shifting each index to its rest wavelength. We elected to derive new wavelength solutions for the 12 spectra (kindly

\footnotetext{
${ }^{2}$ Although circumstantial, these clusters also possess a mean velocity of $-93 \mathrm{~km}^{-1}$. This places them at the lower-velocity end of the M31 GC velocity distribution, which has a maximum-likelihood systemic velocity of $-284 \pm 9$ $\mathrm{kms}^{-1}$, and an overall velocity dispersion of $156 \pm 6 \mathrm{kms}^{-1}$ (Perrett et al. 2002).
} 
provided by J. Cohen and J Blakeslee, see Beasley et al. 2004) by comparing the central index positions in these spectra with known wavelengths from our present M31 data, and Galactic GC data from Puzia et al. (2002). We have also performed a first-order flux calibration to the spectra using the sensitivity functions derived from our long-slit LRIS data using a similar instrumental set-up ${ }^{3}$.

Puzia et al. (2002; henceforth P02) have presented high-quality integrated spectra for 12 Galactic GCs, with the inclusion of a number of bulge clusters. Their sample also contains an integrated spectrum of the outer bulge of the Galaxy. These authors obtained fixed-position (i.e. not drift-scanned) spectra with different pointings (see P02) using the Boller \& Chivens Spectrograph at the ESO $1.52 \mathrm{~m}$ at La Silla. A 600 lines $\mathrm{mm}^{-1}$ grating gave $1.89 \AA$ pixel $^{-1}$, with a fixed 3 arcsec slit yielding a resolution of $\sim 6.7 \AA$.

Four of the GCs in the P02 sample are in common with those in the CBR98 sample, namely NGC 6356, NGC 6528, NGC 6553 and NGC 6624, resulting in a total of 20 unique GCs for our analysis. These four common clusters between the CBR98 and P02 datasets have allowed us to examine any systematic offsets between these data, in addition to estimating the true uncertainties in the CBR98 data (Section 3).

\footnotetext{
${ }^{3}$ In the case of the CBR98 data, we found that the effect on the measured line-strengths using flux- versus un-flux-calibrated spectra was within the measurement uncertainties even for broad indices.
} 


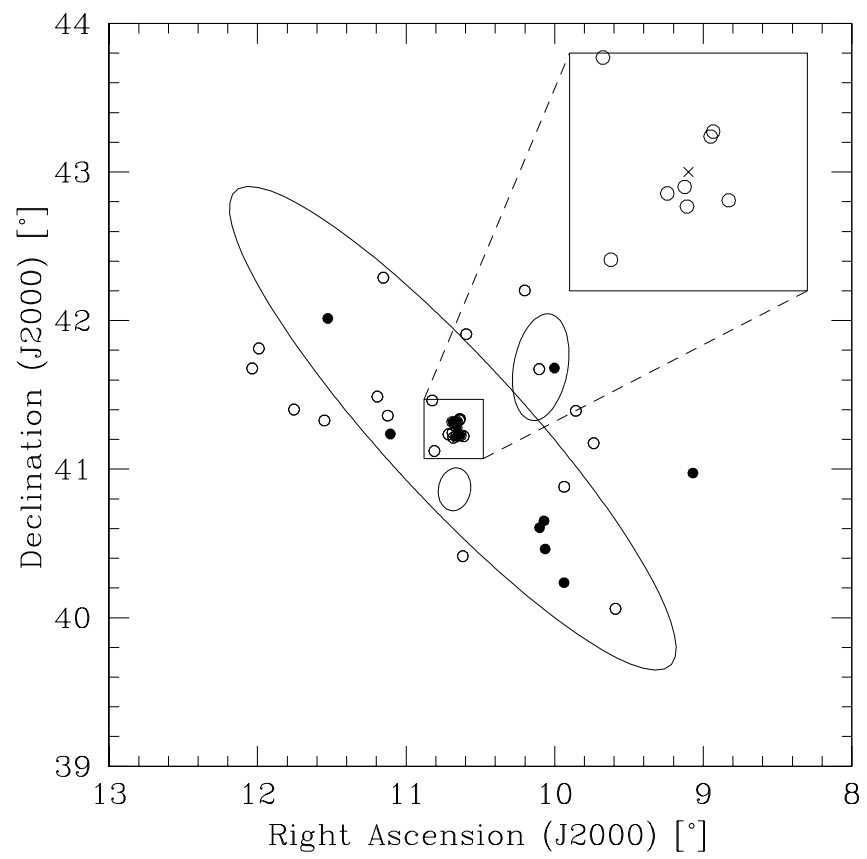

Fig. 1. - Spatial distribution of sample globular clusters in M31. Open circles represent 'normal' globular clusters, filled circles represent clusters which we identify as young ( $<$ few Gyr). Ellipses represent faint isophotes of M31, M32 (lower left) and NGC 205 (upper right). Most GCs have a projected location in the disk/halo of M31, however several are concentrated in the galactic central regions, shown in the enlargement in the upper right corner.

\section{Line-strength Indices}

\subsection{Lick Indices}

We have chosen to investigate the chemical properties of the M31 and Galactic globular clusters using the line-strength indices defined by the Lick group (Burstein et al. 1984; Trager et al. 1998). For the following discussion, we first draw a clear distinction between the Lick indices and the Lick system.

Lick indices now describe a set of 25 indices (see Trager et al. 1998 and Worthey \& Ottaviani 1997 for the latest definitions) which are defined by two central feature bandpasses, and four pseudocontinuum bandpasses (a total of six bandpasses, or wavelengths). However, the Lick system refers to Lick indices which are measured on spectra which match (or mimic) the behavior of the mildly non-linear and un-flux calibrated Lick/Image Dissector Scanner (Lick/IDS). This is achieved by virtue of either the spectra having been acquired with the Lick/IDS, or by calibrating onto the 
Lick/IDS system by matching its resolution and correcting for systematic offsets through observing sufficient Lick standard stars.

The advantages in using the Lick system and indices are that they are widely used by galactic and globular cluster researchers, and that an extensive stellar library exists for the construction of stellar population models (e.g., Worthey et al. 1994). Important drawbacks are that calibrating non-Lick/IDS data onto the Lick system can be difficult, generally requiring many observations of standard stars, and that data of higher resolution than that of the Lick library (8-11 $\AA$ ), must be degraded to this resolution, potentially losing important information.

In principle, line-strength indices measured on flux-calibrated spectra obtained with modern spectrographs and linear detectors should be well reproducible, providing these data are at the same resolution (e.g., Vazdekis 1999). For the case of spectra with good sky-subtraction and flatfielding, no systematic offsets should be observed between measurements of common objects, with the scatter entirely reflecting the random uncertainties between the two observations. Therefore, we have chosen not to correct these data to the Lick system, but compare the CBR98, M31 and P02 data at the Lick resolution. Note for the P02 data, we have 'de-Licked' these indices using the values given in P02. However, for comparison with stellar population models based upon the Worthey et al. (1994) Lick fitting functions, correction to the Lick system is a requirement. We determine these corrections in Section 3.2.

To match the Lick/IDS resolution, the CBR98 and M31 data have been smoothed with a wavelength-dependent Gaussian kernel based on the known variation of FWHM with wavelength of the Lick/IDS given in Worthey \& Ottaviani (1997). These M31 data were then shifted to the rest-frame using the velocities given in Barmby et al. (2000). This shift was unnecessary for the CBR98 data since they were wavelength calibrated in the rest-frame (Section 2.2).

CBR98 originally measured a subset of Lick indices from their spectra using the original index definitions of Burstein et al. (1984). For these re-calibrated CBR98 data, we have measured Lick indices using the updated definitions in Trager et al. (1998) and Worthey \& Ottaviani (1998). Note that the differing grating angles used in these observations (Section 2.2) yielded different spectral ranges, resulting in 6 clusters which do not have measured indices blueward of $4500 \AA\left(\mathrm{C}_{2} 4668\right)$. All the measured Lick indices which fall within the spectral range are given in Table 2.

Due to the high S/N nature of these spectra, photon statistical errors generally underestimate the real uncertainty in the Lick indices measured. Since we have no repeat observations of these clusters with which to determine our repeatability, we have estimated the uncertainty in each index by comparing our measured Lick indices with four GCs which are in common with the P02 sample. We determine the rms difference between each Lick index to yield $\sigma_{\mathrm{rms}}{ }^{4}$. The mean difference and $\sigma_{\text {rms }}$ for each index between the common clusters are listed in Table 3. Unfortunately, only one of

\footnotetext{
${ }^{4}$ In principle, this actually provides an overestimation since we also include the intrinsic uncertainties within the P02 data. However, the quadrature subtraction of these uncertainties in some cases leads to an uncertainty of zero.
} 
the Galactic clusters common between the CBR98 and P02 datasets (NGC 6356) includes all the blue indices in the CBR98 spectrum. Therefore, $\sigma_{\mathrm{rms}}$ for indices $\mathrm{H} \delta_{\mathrm{F}}$ through to Fe4531 was taken to be the absolute difference between the measurement. The shot-noise uncertainties for these data were determined using the method described by Cardiel (1998), which we denote $\sigma_{\mathrm{S}}$, yielding a total error : $\sigma_{\mathrm{T}}^{2}=\sigma_{\mathrm{rms}}^{2}+\sigma_{\mathrm{S}}^{2}$. Values of $\sigma_{\mathrm{T}}$ are also given in alternate rows in Table 2 .

As can be seen, in general the agreement between the two datasets is good and offsets between the CBR98 and P02 data are small. This is true for the most important indices (e.g., CN, $\mathrm{Mg}_{2}$, $\mathrm{Mg}$ b, Fe5270, Fe5335). We see significant offsets in $\mathrm{G} 4300, \mathrm{H} \gamma_{\mathrm{A}}, \mathrm{C}_{2} 4668$ and Fe5015. The reason for these differences is unclear, however we note that the Lick correction employed by P02 for these indices (which we have de-corrected these indices by) are significant.

For the M31 data, twenty-four Lick indices were measured from $\mathrm{H} \delta_{\mathrm{A}}$ (the bluest) to $\mathrm{TiO}_{1}$ (the reddest) as defined in Trager et al. (1998) and Worthey \& Ottaviani (1998). We were unable to measure $\mathrm{TiO}_{2}$ since this index extends beyond our red wavelength limit. These indices are tabulated in Table 4. We have estimated the uncertainties in these data by comparing clusters in common between the two observing runs. There are three clusters in common which are commonly used as velocity standards: 158-213, 163-217 and 225-280 (using the nomenclature of Huchra, Brodie \& Kent 1991), and we have taken the rms, $\sigma_{\mathrm{rms}}$, between index measurements of these clusters as an estimate of our repeatability.

This method worked well for most indices, and any systematic offsets were generally small. The exceptions to this were $\mathrm{H} \delta_{\mathrm{A}}$ and $\mathrm{NaD}$ which both showed rather large offsets between the 1995, December and 1996, September runs $(-0.4 \AA$ and $-0.5 \AA$ respectively) for reasons that remain unclear. In view of this we assigned rms's of $0.4 \AA$ and $0.5 \AA$ respectively, and note that these are problematic indices. Poisson (shot-noise) errors $\left(\sigma_{\mathrm{S}}\right)$ were calculated as for the CBR98 data. Values of $\sigma_{\mathrm{T}}$ are given in alternate rows in Table 4.

\subsection{Corrections onto the Lick/IDS System}

The usual method for correcting to the Lick/IDS system is to observe a range of standard stars in the Lick library which cover the metallicities and range of spectral types of the stellar populations under study (González, 1993). Ideally, these stellar spectra should be obtained at the same time as the program observations, and crucially, obtained using the same instrumental setup. Comparison with the Lick/IDS tabulated values then provides the required corrections (linear or otherwise) in order to mimic the Lick/IDS system.

Unfortunately, no such observations were taken for the CBR98 data, nor for the M31 data. However, there are six Galactic GCs in common between the CBR98 data and Trager et al. (1998), as are three M31 GCs in common between the M31 and Trager at al. (1998) data. The six clusters in common between the CBR98 data and Trager et al. (1998) are NGC 6171, NGC 6205 (M13), NGC 6341 (M92), NGC 6356, NGC 6624 and NGC 6838 (M71). Trager et al. (1998) did not 
measure the higher-order Balmer lines $(\mathrm{H} \delta, \mathrm{H} \gamma)$ for these data, and therefore we have taken the indices measured on the Lick/IDS spectra from Kuntschner et al.(2002). Note that the Galactic and M31 cluster indices given in Trager et al. (1998) were first published in Burstein et al. (1984), with the older Lick indices given in this paper. In order to be consistent with the index bandpass definitions in this study, all our comparisons are performed against the newer measurements given in Trager et al. (1998).

To characterize the relationships between the Lick and CBR98 index measurements, we have used a simple least-squares linear fit; $I_{\text {Lick }}=a+b I_{\mathrm{CBR} 98}$, where $I_{\text {Lick }}, I_{\mathrm{CBR} 98}$ is index in question measured on the Lick and CBR98 data, and the coefficients $a, b$ are given in Table 5 along with the rms of the least-squares fit. We have also calculated the mean difference between these observations (Lick-CBR98) and their respective standard deviations, which provide first-order corrections (i.e., additive corrections) and tabulate them in Table 5. For several of the indices (G4300, Fe4383, Fe4531, $\mathrm{C}_{2} 4668$ and Fe5709) NGC 6171 showed large deviations from the mean, and these were removed from our calculations. We were unable to obtain any corrections for the bluest Lick index $\mathrm{H} \delta_{\mathrm{A}}$.

Comparison between the Trager et al. (1998) and CBR98 data shows significant deviations from unit slope for some indices (Table 5). In some cases, this may be attributable to resolution differences between the spectra (e.g., P02). We tested this by broadening the CBR98 data with wavelength-dependent Gaussian kernels several Angstroms (FWHM) higher and lower than that of the Lick/IDS resolution, and re-measuring Lick indices. In general, we found that we could induce changes in slope of no more than $10 \%$ in these indices with the exceptions of Ca4227 and Fe5335, which are know to be very sensitive to resolution (Vazdekis 1999).

For the M31 data, there are three clusters which are in common with the Trager et al. (1998) sample. This is not sufficient to securely characterize any changes in slope. However these three clusters (158-213, 163-217, 255-280) do populate a reasonable range over the metallicity distribution of M31 clusters (Brodie, Huchra \& Kent give metallicities for these clusters of $[\mathrm{Fe} / \mathrm{H}]^{5}=-1.08 \pm 0.05$, $-0.36 \pm 0.27$ and $-0.70 \pm 0.12$ respectively). Moreover, these three clusters are amongst the highest $\mathrm{S} / \mathrm{N}$ spectra in our M31 sample. In general the agreement between the M31 and Trager et al. (1998) indices is encouraging. We list the linear fit coefficients $a, b, \mathrm{rms}$ of the fit, mean values of (Lick-M31) and the standard deviations for each index in Table 6.

\section{Analysis of the Main Sample}

For the remainder of this paper, we use the indices given in Tables 2 and 4, uncorrected to the Lick system. Where there are clusters common between the CBR98 and P02 Galactic data, we have used the P02 data due to their more reliable error estimates.

\footnotetext{
${ }^{5}$ Unless stated otherwise, $[\mathrm{Fe} / \mathrm{H}]$ refers to metallicities on the Zinn \& West (1984) scale.
} 


\subsection{Balmer Lines}

Spinrad \& Schwiezer (1972), Rabin (1981) and later Burstein et al. (1984) found evidence that that M31 GCs were enhanced in the Balmer lines with respect to their Galactic counterparts ${ }^{6}$. Specifically, Burstein et al. (1984) found that at a given $\mathrm{Mg}_{2}$ linestrength, the $\mathrm{H} \beta$ index was on average higher than that of the Galactic globular clusters at $\mathrm{Mg}_{2}>0.1$. These authors explored three possible origins for these differences-contributions from hot horizontal branch stars, blue stragglers or younger ages-and favored this latter interpretation.

Subsequent work by Brodie \& Huchra $(1990,1991)$ and Huchra et al. (1991) with a more extensive sample indicated that only a subset of M31 clusters appeared to have such enhanced $\mathrm{H} \beta$, and indeed this question still remains controversial. We now examine this issue with the Galactic and M31 GC data.

\footnotetext{
${ }^{6}$ Note that although Rabin (1980) and Burstein et al. (1984) reached similar conclusions regarding Balmer-line enhancements, in detail their respective $\mathrm{H} \beta$ measurements show very poor agreement.
} 

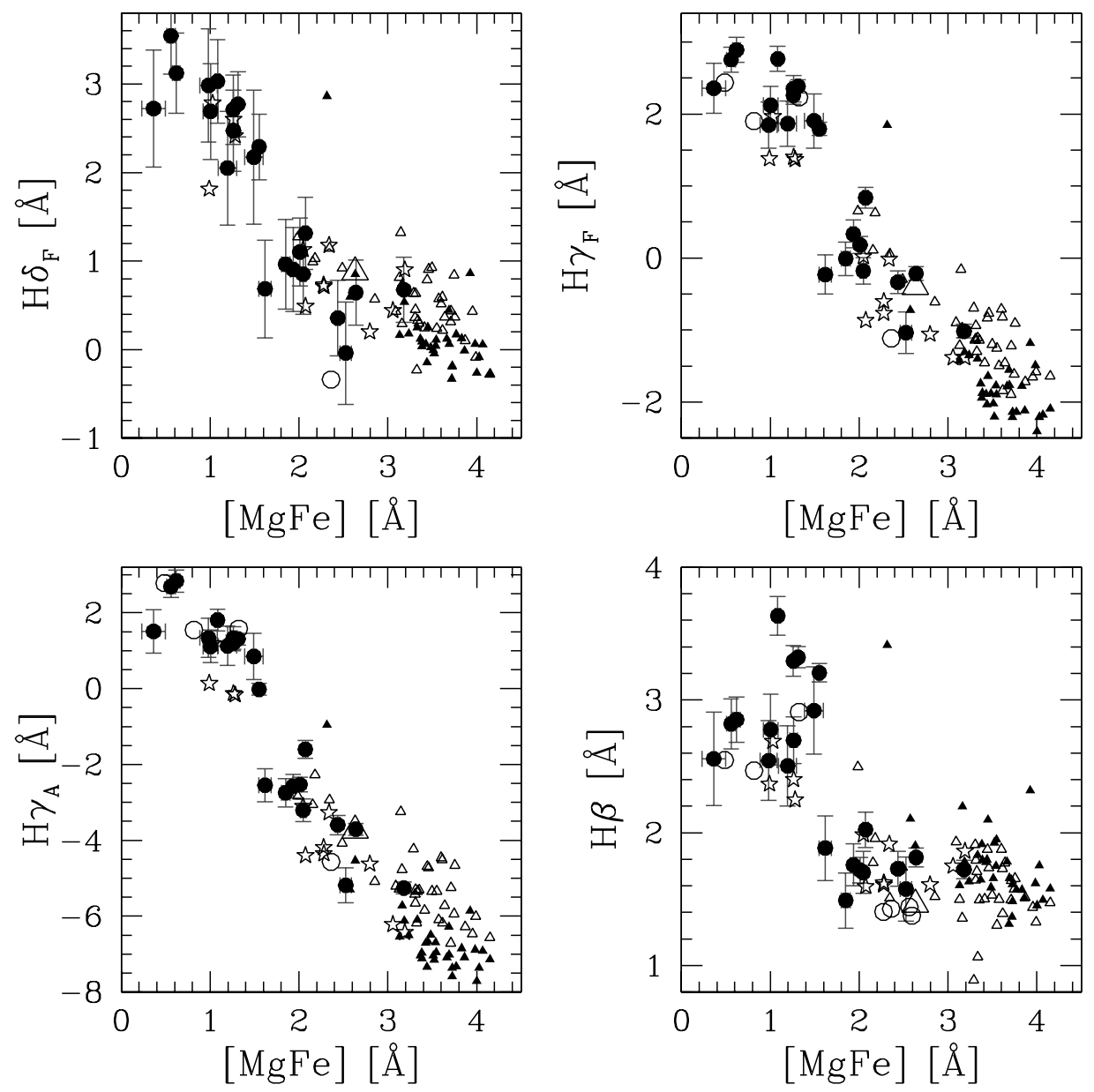

Fig. 2.- Comparison between the M31 data (filled circles) and Galactic GC data (open circles:CBR98; stars:P02) for the $\mathrm{H} \beta, \mathrm{H} \gamma$ and $\mathrm{H} \delta$ Lick indices at a given [MgFe]. Uncertainties (grayed error bars) are only shown for M31 data for the sake of clarity. The open triangle indicates the outer Galactic bulge observations of P02. Small open and filled triangles represent data for Coma and field elliptical galaxies (Sánchez-Blázquez 2003). For the metal-rich M31 GCs, we see no significant enhancement in the Balmer lines compared to the Galactic data.

In Figure 2 we plot the Lick Balmer indices of CBR98 and P02 compared to the M31 data at given value of $[\mathrm{MgFe}]^{7}$. We use $[\mathrm{MgFe}]$ since it is metallicity sensitive, relatively insensitive to abundance ratio variations (González 1993; Thomas, Maraston \& Bender 2003), and the narrow indices Mg b, Fe5270, and Fe5335 are not particularly susceptible to systematic offsets between data.

${ }^{7}[\mathrm{Mg} b \times(\mathrm{Fe} 5270+\mathrm{Fe} 5335)]^{1 / 2}$, González (1993) 
The first thing to note in the four panels of Figure 2 are the discontinuities in the plots at $[\mathrm{MgFe}] \sim 1.6$. These gaps in the index-index relations reflect the well-established bimodality of the Galactic and M31 cluster systems, a gap which is not particularly evident in the Trager et al. (1998) data. The presence of these discontinuities reflects the high $\mathrm{S} / \mathrm{N}$ of these present data. To contrast the Balmer indices of these data, we find it convenient to separate the clusters into two groups, $[\mathrm{MgFe}]<1.5$, and $[\mathrm{MgFe}] \geq 1.5$. Using the empirical $[\mathrm{MgFe}]-[\mathrm{Fe} / \mathrm{H}]$ relation derived by $\mathrm{P} 02,[\mathrm{MgFe}]=1.5 \AA$ corresponds to $[\mathrm{Fe} / \mathrm{H}] \sim-0.8$. We refer to these groups as the 'low-metallicity' and 'high-metallicity' groups respectively.

Inspection of Figure 2 shows no evidence of systematic enhancements in $\mathrm{H} \beta, \mathrm{H} \gamma$ or $\mathrm{H} \delta$ between the high-metallicity M31 and Galactic GC data. Furthermore, there is no evidence of a significant change in slope in any of the index-index plots which was seen by Burstein et al. (1984). Whilst there does appear to be a mild enhancement in several clusters at $[\mathrm{MgFe}] \sim 2$, which is most pronounced in their $\mathrm{H} \gamma_{\mathrm{A}}$ and $\mathrm{H} \gamma_{\mathrm{F}}$ indices, this is not particularly significant since there are two Galactic GCs (NGC 6388 and NGC 6637) at this metallicity which differ by $\sim 0.4 \AA$ in $\mathrm{H} \beta$; NGC 6388 lies squarely amongst these M31 clusters. The mean values of the $\mathrm{H} \beta$ indices for all the high metallicity M31 and Galactic clusters (P02 and CBR98 data) is $1.75 \AA \pm 0.14 \AA$ and $1.63 \AA$ $\pm 0.20 \AA$ respectively. The straight mean, however, is not the fairest comparison in this case since the slope of $[\mathrm{MgFe}]-\mathrm{H} \beta$ is non-zero, and there are relatively more M31 clusters at $[\mathrm{MgFe}] \sim 2$ than there are Galactic clusters. The mean $\mathrm{H} \beta$ of solely the metal-rich P02 data yields $1.74 \pm 0.16 \AA$ which is identical to that of the metal-rich M31 GCs.

Turning our attention to the metal-poor clusters, the M31 GCs do appear to have somewhat stronger $\mathrm{H} \beta$ and $\mathrm{H} \gamma_{\mathrm{F}}$ indices. The mean $\mathrm{H} \beta$ for these metal-poor M31 clusters is $2.95 \AA \pm 0.35 \AA$, whereas that of the metal-poor Galactic clusters is $2.52 \AA \pm 0.22 \AA$. This difference of $0.43 \AA$ in $\mathrm{H} \beta$ is close to the dispersion in the metal-poor M31 clusters alone. Such variations are not particularly unusual amongst metal-poor globular clusters, and possibly arise from differences in horizontal branch morphology (Lee et al. 2000; P02).

In Figure 2 we have also plotted the high-quality data for Coma and Field ellipticals from Sánchez-Blázquez (2003). Their sample consists of 59 galaxies from the field and Virgo cluster, and a further 34 galaxies from the central regions of the Coma cluster. These galaxies cover an absolute magnitude range $-22.5<M_{B}<-16.5$, and comprise of dwarf to giant elliptical galaxies. All the observations were performed with an $\mathrm{R}_{e} / 4$ aperture and fully corrected to the Lick system. We have 'de-corrected' these data (using the offsets kindly supplied by P. Sánchez-Blázquez) in order directly compare with our data.

Interestingly, there appear to be obvious differences between the loci of the field and Coma cluster galaxies with respect to the GC data. The field galaxies generally appear to have lower Balmer line-strengths when compared to the cluster galaxies at a given [MgFe]. Moreover, there is a suggestion that the locus of the GC data more closely follows that of the field galaxies than of those in clusters. A straightforward (but non-unique) interpretation is that the GCs and field 
ellipticals are generally older than cluster galaxies. We note that the metallicities (as given by $[\mathrm{MgFe}])$ of the most metal-rich M31 and Galactic GCs do not quite form a continuous relation with the galaxies. However, as noted by P02, the metallicities of the most metal-rich Galactic GCs are comparable to the integrated Milky Way bulge light. This is consistent with the idea that more metal-rich galaxies harbor more metal-rich GC systems (van den Bergh 1975; Brodie \& Huchra 1991; Strader, Brodie \& Forbes 2004).

We see no difference in the Balmer-lines of the metal-rich Galactic and M31 GCs at a given value of $[\mathrm{MgFe}]$, whereas previous studies (Burstein et al. 1984; Brodie \& Huchra 1991) have documented possible differences (at a given value of $\mathrm{Mg}_{2}$ ). What is the origin of this disagreement between studies? One possible answer is $\mathrm{S} / \mathrm{N}$; these present data represent an improvement in $\mathrm{S} / \mathrm{N}$ over the older studies. For example, the mean uncertainty in our $\mathrm{H} \beta$ measurements are $0.17 \AA$, whereas in the Burstein et al. (1984) study it is $0.26 \AA$. However, the effect of larger errors in e.g., $\mathrm{H} \beta$ should in principle act to wash out any systematic differences, rather than enhance them. As shown in Table 3 the agreement between the P02 and CBR98 data for the indices in question is excellent (with the possible exception of $\mathrm{H} \gamma_{\mathrm{A}}$, however in this case there is only one common cluster between these datasets). For example, the mean difference in the $\mathrm{H} \beta$ index for the four clusters in common is only (P02-CBR98) $-0.0027, \sigma=0.13 \AA$.

Indeed, in comparison with the CBR98 and P02 data, the $\mathrm{H} \beta$ indices from Trager et al. (1998) for their Galactic GC data look systematically low. This is also evident when these data are compared to stellar population models (Beasley et al. 2000; Kuntschner et al. 2003). For the three clusters in common between the P02 data and Trager et al.(1998), the difference is (P02-Trager) $0.44 \pm 0.28 \AA$. By comparison, the mean difference in $\mathrm{H} \beta$ between the M31 and Galactic clusters with $\mathrm{MgFe}>1.5$ in Trager et al. (1998) is $\sim 0.6 \AA$, which lead Burstein et al. (1984) to conclude that the M31 GCs may exhibit $\mathrm{H} \beta$ enhancement.

It is hard to see how these differences have arisen, since the $\mathrm{H} \beta$ index is only weakly affected by continuum slope changes or resolution differences. Experiments involving adding synthetic noise to our spectra, showed no signs of deviations from Gaussian errors. One possibility is foreground contamination from disk and/or halo stars, however this is not expected to be a significant effect (P02) and is unlikely to occur for all the metal-rich objects in question. Other possibilities include uncertain sky subtraction especially in areas of high background (CBR98, P02) and variations in the sampled luminosity of the clusters (P02). Based upon their thorough analysis of these issues, we prefer the P02 indices over previous measurements.

In any event, on the basis of these present data, we conclude that there is no Balmer-line enhancement in this sample of M31 globular clusters when compared to their Galactic counterparts at high metallicity $([\mathrm{Fe} / \mathrm{H}] \geq-0.8)$. At low metallicity, there is some evidence for stronger $\mathrm{H} \beta, \mathrm{H} \gamma$ in the M31 clusters which is perfectly consistent with horizontal branch variations. 


\section{2. $\quad \mathrm{C}$ and $\mathrm{N}$ in Globular Clusters}

One of the principle results of the Burstein et al. (1984) study was the identification of strong $\mathrm{CN}$ molecular bands $(\lambda 4150 \AA)$ in the spectra of M31 clusters. They found that at a given $\mathrm{Mg}_{2}$ index strength, the $\mathrm{CN}$ feature in the M31 clusters was up to 0.1 mag stronger than that seen in elliptical galaxies. However, no convincing comparison could be made with the Galactic GCs due to the lack of the most metal-rich objects in their sample. A similar result was found by Brodie \& Huchra (1991) using a similar definition of the near-UV cyanogen feature.

The lack of any significant variation in the G-band $(\mathrm{CH})$ feature was indicative in both studies that $\mathrm{C}$ was not the main culprit in the CN-enhancement. Subsequent data, with the inclusion of the bluer NH feature at $\lambda 3360 \AA$, (Tripicco 1989, Ponder et al. 1998; Li \& Burstein 2004) strongly suggests the presence of enhanced $\mathrm{N}$ in the integrated spectra of M31 clusters compared to Galactic dwarfs of comparable spectral type.

However, the Galactic globular cluster samples of Burstein et al. (1984), and Brodie \& Huchra (1991) did not include the most metal-rich Galactic GCs in which one may expect to see the strongest CN enhancements. Fortunately the P02 data provide such a sample of clusters, and

indeed these authors have noted that the CN strengths of the most metal-rich Galactic GCs are significantly enhanced when compared to the integrated spectrum of the (outer) Galactic bulge. Moreover, these authors also commented that these most-metal-rich Galactic GCs in their sample have similar CN strengths to the M31 sample of Trager et al. (1998). 


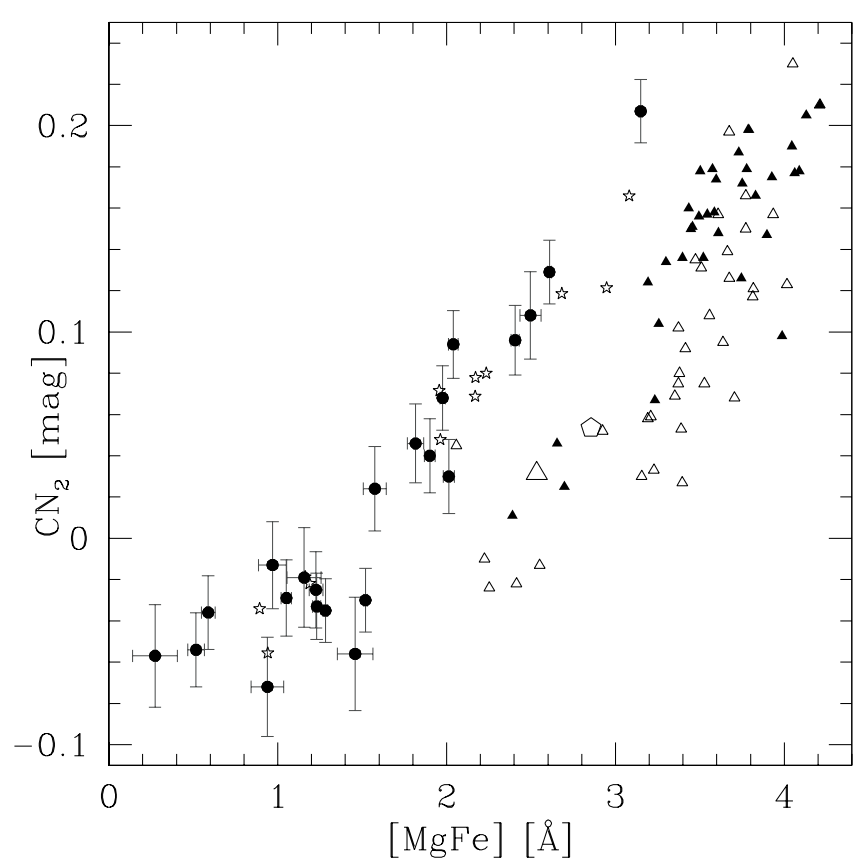

Fig. 3. - The Lick $\mathrm{CN}_{2}$ index as a function of $[\mathrm{MgFe}]$ for the M31 GCs (solid circles with error bars), and P02 Galactic GC data (open stars). The open triangle represents the mean Galactic bulge measurement from P02, and the pentagon denotes the M31 nuclear measurement of Trager et al. (1998). Small filled and open triangles represent data for field and Coma elliptical galaxies respectively (Sánchez-Blázquez et al. 2003).

In Figure 3 we show the Lick $\mathrm{CN}_{2}$ index, for the M31 and Galactic GC data, at a given [MgFe]. We show the $\mathrm{CN}_{2}$ index rather than $\mathrm{CN}_{1}$, since this latter index suffers from contamination from the adjacent $\mathrm{H} \delta$ feature. Note that the CBR98 data generally do not reach down to $\sim 4000 \AA$, and therefore we rely on the P02 dataset for comparison to these M31 data. Figure 3 suggests that the CN indices for the Galactic and M31 clusters are all significantly enhanced with respect to the bulges of the Milky Way and M31. This is consistent with the findings of Burstein et al. (1984) and P02. The difference in $\mathrm{CN}_{2}$ between the Milky Way outer bulge observations and the M31 and P02 data at the same value of $[\mathrm{MgFe}]$ is $\sim 0.07$ mag.

We have also plotted $\mathrm{CN}_{2}$ data from the field and cluster sample of ellipticals from SánchezBlázquez et al. (2003). As discussed by these authors, the $\mathrm{CN}_{2}$ indices of the field and Coma cluster ellipticals appear to show different behavior; field ellipticals appear more enhanced in CN than those in the denser environment of Coma. Indeed, the locus of the field galaxies is closer to that of the GCs, although there still appears to be a substantial offset between the two. SánchezBlázquez et al. (2003) concluded that both $\mathrm{N}$ and $\mathrm{C}$ were enhanced in the field relative to cluster ellipticals, possibly through pollution of the ISM through low- and intermediate-mass stars. 
We see no strong evidence for an enhancement in $\mathrm{CN}_{2}$ in the M31 clusters with respect to the Galactic GCs at any metallicity. Similar behavior is seen in the $\mathrm{CN}_{1}$ index. This is a rather surprising result, considering the fact that the CN enhancement in M31 clusters has been well documented (Burstein et al. 1984; Tripicco 1989; Davidge 1990; Tripicco \& Bell 1990; Brodie \& Huchra 1991; Ponder et al. 1998; Li \& Burstein 2003). Our findings do not appear to be an artifact of not correcting to the Lick system, which actually exacerbates the problem since the correction is significant ( $0.032 \mathrm{mag}$ for $\mathrm{CN}_{2}$ ). Through experimentation, we find that both $\mathrm{CN}_{1}$ and $\mathrm{CN}_{2}$ can vary by up to several tenths of a magnitude depending upon the details of our flux calibrations, but this is generally only true for the very metal-poor clusters which have relatively large amounts of blue flux. This problem is particularly acute in the blue part of the spectrum for broad indices, since the throughput of the 'pre-blue-arm' LRIS roles off rapidly at $\sim 4000 \AA$.

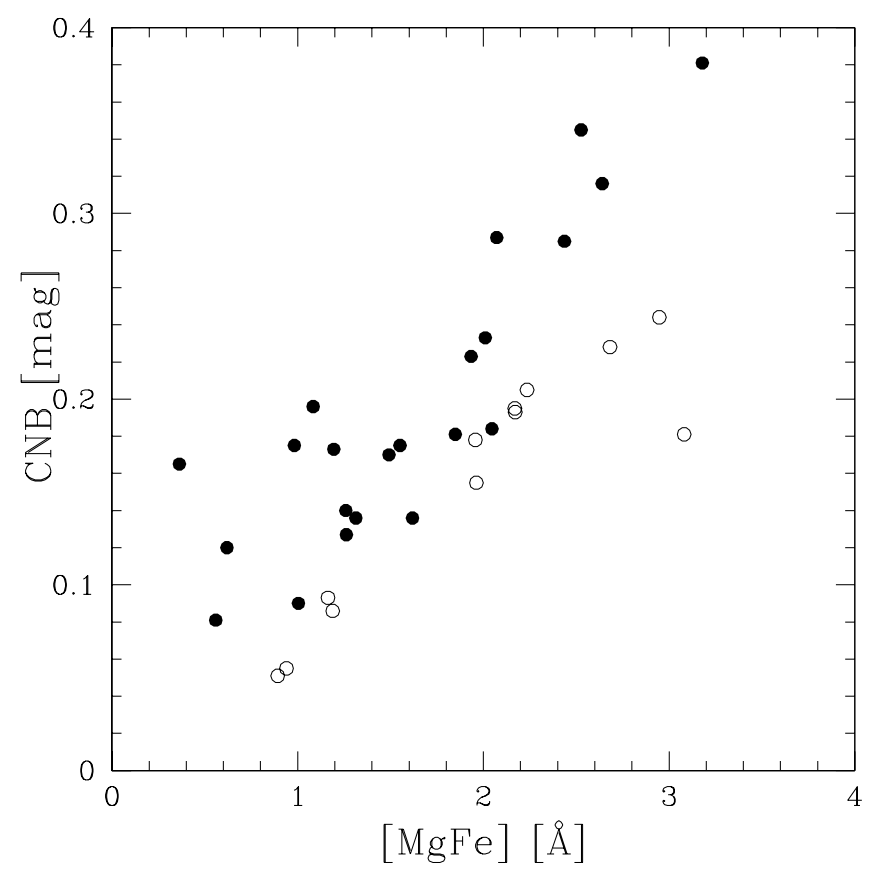

Fig. 4. - The strength of the blue cyanogen index (CNB) at a given $[\mathrm{MgFe}]$ for our M31 data (filled symbols) and the Milky Way GC data of P02. The M31 GCs appear clearly enhanced with respect to Galactic GCs at all metallicities.

We have, however, measured the near-UV cyanogen index at $\lambda 3883 \AA$ (using the definitions given in Brodie \& Huchra 1990) for the M31 and P02 data, which through experimentation appears to be much less sensitive to our flux-calibration uncertainties ${ }^{8}$. The $\mathrm{CN}$ index at $\lambda 3883 \AA$ is also

\footnotetext{
${ }^{8}$ The truth of this statement is both data-dependent, and index-definition dependent. For example, the singlesided $\lambda 3883 \AA$ index often used in stellar studies is prone to significant zero-point offsets (e.g.Cohen, Briley \&Stetson
} 
significantly stronger than the Lick CN indices.

The strength of CNB with $[\mathrm{MgFe}]$ is plotted in Figure 4. The difference in CNB between the M31 and Galactic GCs is startling, and is evident at all metallicities. The metallicity range in which we can perform a direct comparison between the M31 and Galactic GCs is defined by the P02 data, with $-1.48 \leq[\mathrm{Fe} / \mathrm{H}] \leq-0.34$. At $[\mathrm{Fe} / \mathrm{H}] \sim-1.5$, the mean difference between the Galactic and M31 data is $\Delta \mathrm{CNB} \sim 0.1$ mag. For the most metal-rich GCs at $[\mathrm{Fe} / \mathrm{H}] \sim-0.4, \Delta \mathrm{CNB} \sim 0.2 \mathrm{mag}$.

To check the reality of this result, since Lick $\mathrm{CN}_{2}$ and $\mathrm{CNB}$ appear to disagree, we have taken one of the 'CN-weak' Galactic GCs, NGC 6441, the spectrum of which was kindly supplied by T. Puzia, and divided it by one of the 'CN-strong' M31 clusters (225-280). These clusters have very similar $[\mathrm{MgFe}]$ strengths (i.e., very similar metallicities), and care was taken to ensure these spectra were at the same normalization, resolution and spectral sampling. This difference spectrum is shown in Figure 5.

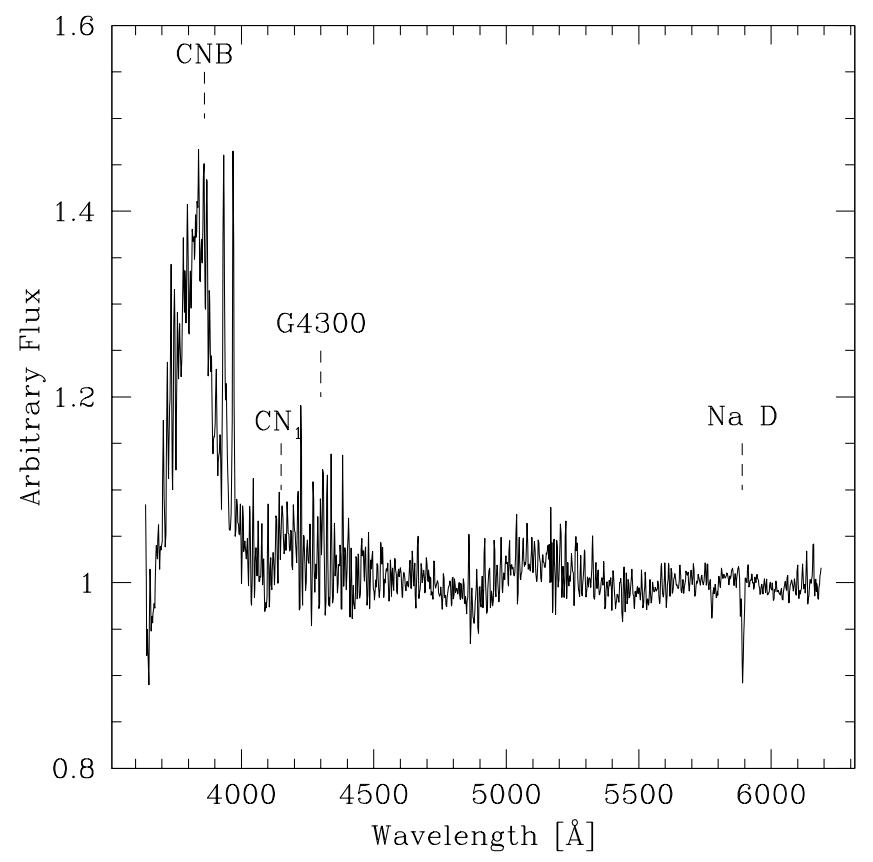

Fig. 5.- Residual spectrum after the spectrum of NGC 6441, a Galactic GC, was divided by that of 225-280, a GC in M31. Both these clusters have $[\mathrm{Fe} / \mathrm{H}] \sim-0.5$. The absorption system at CNB is clearly enhanced, indicating greater CN absorption in the M31 GC. The Na D residual probably reflects the contribution of interstellar sodium absorption in the spectrum of the Galactic GC.

CNB in the residual spectrum is clearly enhanced, indicating that the M31 GC has significantly

2002). 
stronger CN absorption at the same metallicity than NGC 6441. Weak enhancement is also seen in the Lick CN index, an enhancement which is washed out at lower metallicities. G4300 also appears to be mildly enhanced. Since this index measures $\mathrm{CH}$, this would suggest that $\mathrm{C}$ is also mildly enhanced (see below).

On the basis of the clear differences in the CNB index that we observe, we conclude that CN is enhanced in the M31 GCs with respect to their Galactic counterparts at all metallicities. The lack of obvious enhancement in the $\mathrm{CN}_{1}$ and $\mathrm{CN}_{2}$ indices between the Galactic and M31 clusters probably reflects the relatively low sensitivities of these indices to $\mathrm{CN}$, when compared to CNB, rather than any systematic offset between these data (see Trager (2004) for a discussion of this issue).

Variations in $\mathrm{CN}$ are thought to be controlled largely by variations in $\mathrm{N}$, since free $\mathrm{C}$ is rapidly incorporated into the $\mathrm{CO}$ molecule. Therefore, $\mathrm{CN}$ 'enhancement' is believed to reflect an enhancement in N, this picture is supported through observations of the NH molecule (Ponder et al. 1998; Li \& Burstein 2004). However, can we rule out carbon variations? Two Lick indices primarily sensitive to $\mathrm{C}$ are the $\mathrm{C}_{2}$ swan band $\mathrm{C}_{2} 4668$ (formerly Fe4668) and $\mathrm{G} 4300$ which measures $\mathrm{CH}$.

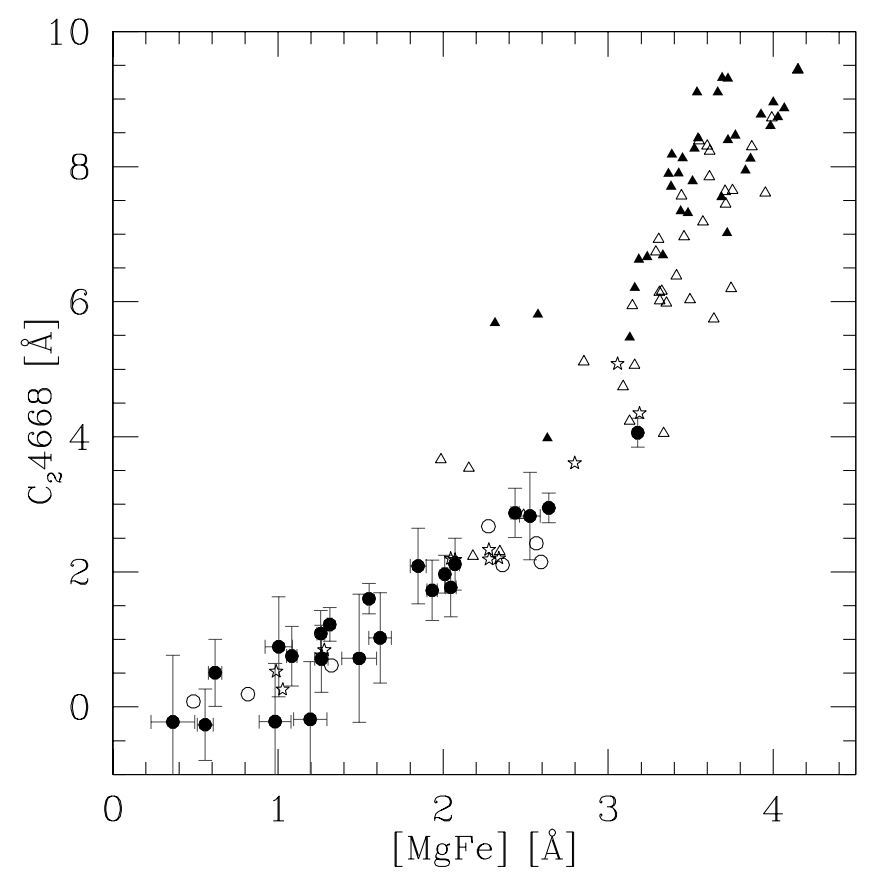

Fig. 6. - The Lick $\mathrm{C}_{2} 4668$ index as a function of [MgFe]. Symbols are the same as for Figure 3. 


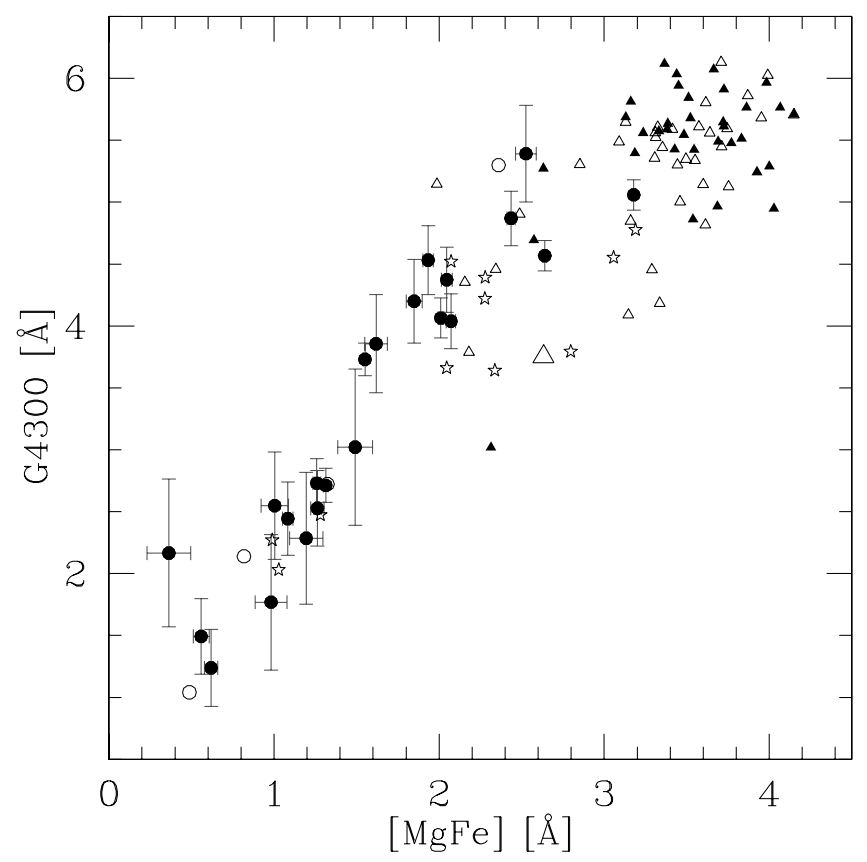

Fig. 7.- The G4300 (CH) index as a function of $[\mathrm{MgFe}]$. Symbols are the same as for the previous figure.

In Figure 6 we plot $\mathrm{C}_{2} 4668$ as a function of $[\mathrm{MgFe}]$. These data define a very tight sequence in this plot, with a clear change of slope at $[\mathrm{MgFe}] \sim 3$, the transition from GCs to galaxies. On the basis of Figure 6, we see no evidence for differences in $\mathrm{C}$ amongst the GCs. However, as discussed by Sánchez-Blázquez et al. (2003), this index is enhanced in field ellipticals with respect to cluster ellipticals at a given metallicity. The behavior of the other carbon sensitive index, G4300, is plotted in Figure 7. In this case, there is a suggestion that at $[\mathrm{MgFe}]>2$, the M31 GCs do have a stronger G4300 indices than the Galactic GCs. The M31 clusters also have significantly stronger G4300 than the outer bulge observations of the Milky Way. Unfortunately, the interpretation of such variations in G4300 are complicated by the fact that this index is also sensitive to age and the $\mathrm{O}$ and $\mathrm{Ti}$ abundances.

\section{Young Star Clusters in M31}

Eight of the initial 42 GC candidates in the M31 sample exhibit mid-A to early F-type spectra, as would be expected of A-/F-stars or young star clusters. The existence of very young clusters in the M31 system has been mentioned previously; Huchra, Brodie \& Kent (1991), and Barmby et al. (2000). 
One of these clusters, 324-051, is also designated Hubble V in NGC 205, and was taken as a reference template for the other candidate young clusters in M31. Hubble V was identified by Da Costa \& Mould (1988) as being a young object from $3 \AA$ resolution optical integrated spectra. Based on its similarity to the Small Magellanic Cloud cluster NGC 419, Da Costa \& Mould (1988) concluded that Hubble $\mathrm{V}$ was 1 Gyr old, but could not rule out ages several Gyr older than this, or as young as 100 Myr.

From the broad Balmer lines, weak metal-lines and relatively blue continua of the cluster spectra in our sample, we assign the clusters spectral types A5-F0. Such $\sim 2 \mathrm{M}_{\odot}$ stars which dominate the main-sequence turn-off will complete core-hydrogen burning after approximately 1 Gyr (Schaller et al. 1992), consistent with the age of Hubble V found by Da Costa \& Mould (1988)

The spectra of these cluster candidates are shown in Figure 8. Clear evolution in several indices can be seen as we proceed to later types in the figure; the Balmer lines all weaken, the ratio of $\mathrm{Ca}$ II $\mathrm{K} / \mathrm{Ca}$ II $\mathrm{H}+\mathrm{H} \epsilon$ increases as metal-lines strengthen and $\mathrm{H} \epsilon$ weakens, and the G-band (G4300 in the Lick definition) which measures the $\mathrm{CH}$ molecule starts to appear in the Hubble $\mathrm{V}$ spectrum and later types. The slope of the spectra also noticeably change, from bluer to redder, although reddening also seems to be playing a role here (e.g., 321-046). The clusters 222-227 displays a rather unusual shape, possibly due to heavy reddening, leading to its 'peculiar' designation in Table 1. It also happens to be our lowest $\mathrm{S} / \mathrm{N}$ spectrum. 


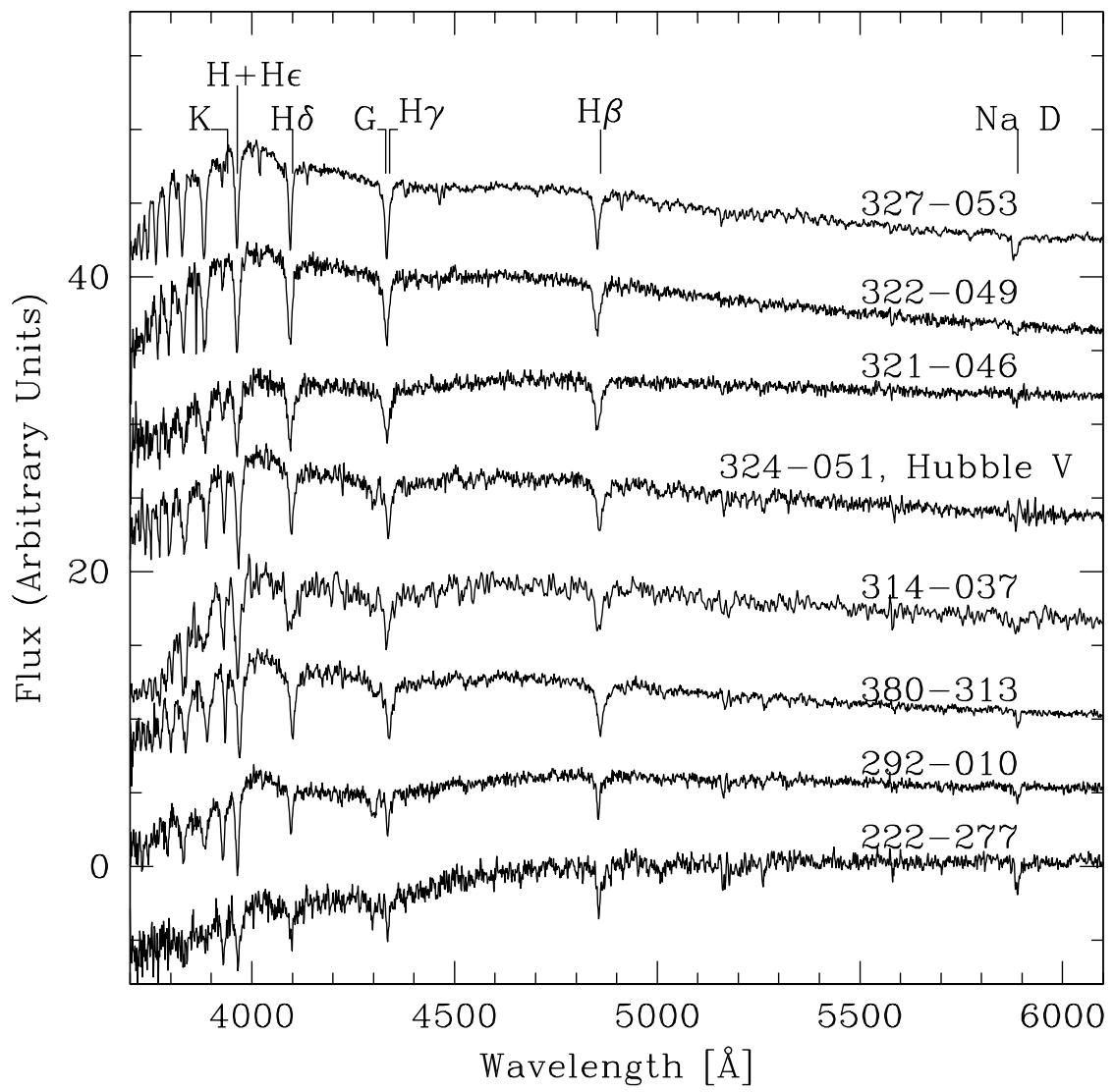

Fig. 8. - LRIS spectra at $\sim 5.6 \AA$ resolution for the eight candidate young globular clusters in the M31 sample. The spectra have been normalized, with additive constants on the $y$-axis for display purposes. The spectra are arranged in approximate order of spectral type, running from earlier (A5) to later types (F0; top to bottom).

The first obvious question to ask of these young cluster candidates is : are these really globular clusters belonging to M31? In the original Bologna group catalogue (Battistini et al. 1987), which was based on photographic plates, all the clusters for which we have spectra are designated A- or B-classes, i.e., high-confidence clusters, on the basis of their non-stellarity and circularly symmetric brightness distributions. This effectively rules out that these objects are foreground Galactic blue horizontal branch stars, or blue stragglers.

As evidenced by the Large Magellanic Cloud (LMC) system of star clusters, the separation of open and globular clusters is not always clear (e.g., Hodge \& Wright 1967). However, the distinction made is usually one of mass and compactness. The absence of high-resolution imaging for all the 
candidates precludes the secure identification of these objects ${ }^{9}$. However, HST/WFPC2 imaging does exist for Hubble V (cycle 6, proposal ID 6699, PI D. Geisler). We show the PC chip image of this object in the F814W filter in Figure 9. The cluster is clearly resolved into stars, and Kim et al. (2002) have measured surface brightness profiles for this cluster finding that it is better fit by a King (1966) model than a power a law which is usually applicable for open clusters (Elson, Fall \& Freeman 1987). Kim et al.'s derived concentration parameter of $c=0.5$ is comparable to the small Milky Way cluster AM-4 (Madore \& Arp 1982). The mass to light ratios of young clusters evolve rapidly due to the short main-sequence lifetimes of massive stars, this in turn strongly effects any mass estimates for the clusters. We return to estimating the cluster masses later when we have more secure age determinations for these objects.

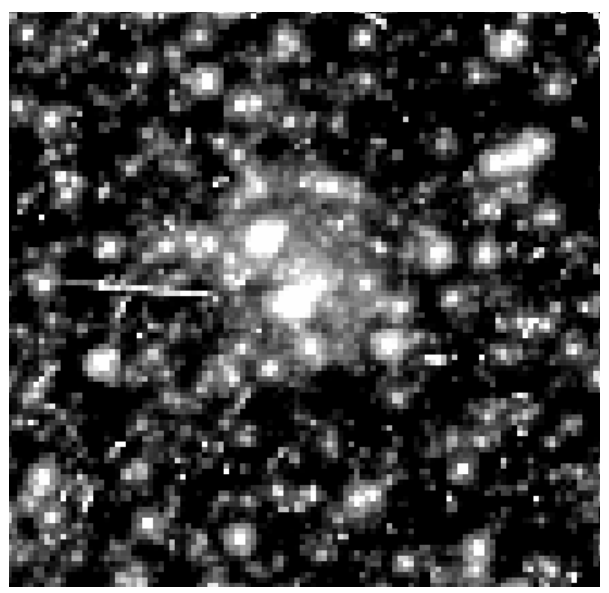

Fig. 9.- HST/PC F814W image of the young cluster in NGC 205, Hubble V. The image is $5^{\prime \prime}$ across, which, at the distance of M31, corresponds to a physical diameter of $\sim 17 \mathrm{pc}$. The pixel scale of the PC chip is $0.045 \mathrm{arcsec} / \mathrm{pixel}$.

Age-dating young stellar populations $(<1$ Gyr) from integrated spectra presents a different set of challenges from older stellar systems. For stellar populations older than $\sim 2 \times 10^{8} \mathrm{yr}$, Balmer lines decrease monotonically with time and may be used to estimate the age of the system (e.g. Bruzual \& Charlot 2003). The key difficulty lies in determining the metallicity of the stellar population, since metal lines become very weak in young objects (e.g., Larsen \& Brodie 2002). We have taken two approaches: first we compare the M31 spectra with a series of LMC templates (Beasley et al. 2002) which bracket the expected age range, and then use stellar population models to provide independent age and metallicity information on the clusters. The two approaches are complementary, and provide useful insight into the reliability of each method.

The LMC cluster system consists of clusters with a range of metallicities and ages, from very

\footnotetext{
${ }^{9}$ We are currently pursuing such imaging for these objects
} 
metal-poor 'classical' globular clusters to intermediate-aged and young, near-solar metallicity star clusters (e.g., Olsen et al. 1998; Geisler et al. 2003; Piatti et al. 2003) ${ }^{10}$. This range of cluster types provides an excellent source of templates for more distant studies (Leonardi \& Rose 2003). Beasley et al. (2002) published integrated spectra of 24 LMC star clusters with 'SWB-types' (Searle, Wilkinson \& Bagnuolo 1980; hereafter SWB) ranging from IVA-VII. Whilst the exact agecalibration of the SWB system is still a source of uncertainty, using the calibration of Bica, Claria \& Dottori (1992), these types correspond to ages 0.20 16 Gyr. The earlier calibration of Cohen (1982) gives somewhat older ages for the intermediate SWB-types (III-VI).

Spectra for SWB I-III clusters (0.01-0.20 Gyr) were also obtained by Beasley et al. (2002), but remained unpublished since the stellar population models assessed did not extend to such young ages. We have used the full sample of Beasley et al. (2002) which consists of 65 clusters, the majority of which are less than 1 Gyr old. In order to obtain the best match between the young M31 clusters and the LMC templates, we cross-correlate each M31 spectrum against the entire LMC library, and select the LMC cluster which yields the highest (normalized) cross-correlation peak height. For this purpose, the M31 spectra were smoothed and re-sampled to match the natural resolution of the LMC data $(6.5 \AA)$. The spectra were then normalized by a low order polynomial (since the LMC data are not flux calibrated), and cross-correlated in the wavelength interval 4000-5400 $\AA$, which is the effective wavelength range of the LMC data.

To test the method, we first cross-correlated the M31 cluster 324-051 (Hubble V) against the LMC library, since we have some independent age information for this cluster. Da Costa \& Mould (1988) determined an age of $\sim 1$ Gyr for this cluster, with a lower bound of $100 \mathrm{Myr}$, whilst Lee (1996) determined from his measured color of the cluster $\left(\mathrm{B}-\mathrm{V}_{0}=0.33\right)$ that the cluster is closer to 300 Myr. The best correlation template we obtain for Hubble V is NGC 1940, an SWB II cluster with $\mathrm{B}-\mathrm{V} \sim 0.3$ (Bica et al. 1992). SWB II corresponds roughly to ages 30-70 Myr, which is surprisingly young.

The normalized spectra of Hubble V and NGC 1940 are shown in Figure 10. The two clusters look rather similar, and this is supported by examination of the residual spectrum (dividing the Hubble V spectrum by that of NGC 1940). Apart from the mild 'P-Cygni' profile near $\mathrm{H} \gamma$, the residuals are relatively modest. There also appears to be a slight enhancement in the CN residuals, in the sense that $\mathrm{CN}$ is lower in Hubble $\mathrm{V}$, although the reality of this is unclear at present.

\footnotetext{
${ }^{10}$ The age-metallicity relation for the LMC clusters in not continuous. It has been known for some time that there is an 'age-gap' between 3 10 Gyr (e.g., Jensen, Mould \& Reid 1988) with a corresponding 'metallicity gap' at [Fe/H] $\sim-1$ (Olszewski et al. 1991). This, however, is not a concern for the present study since we are interested in LMC cluster templates which are younger than a few Gyr.
} 


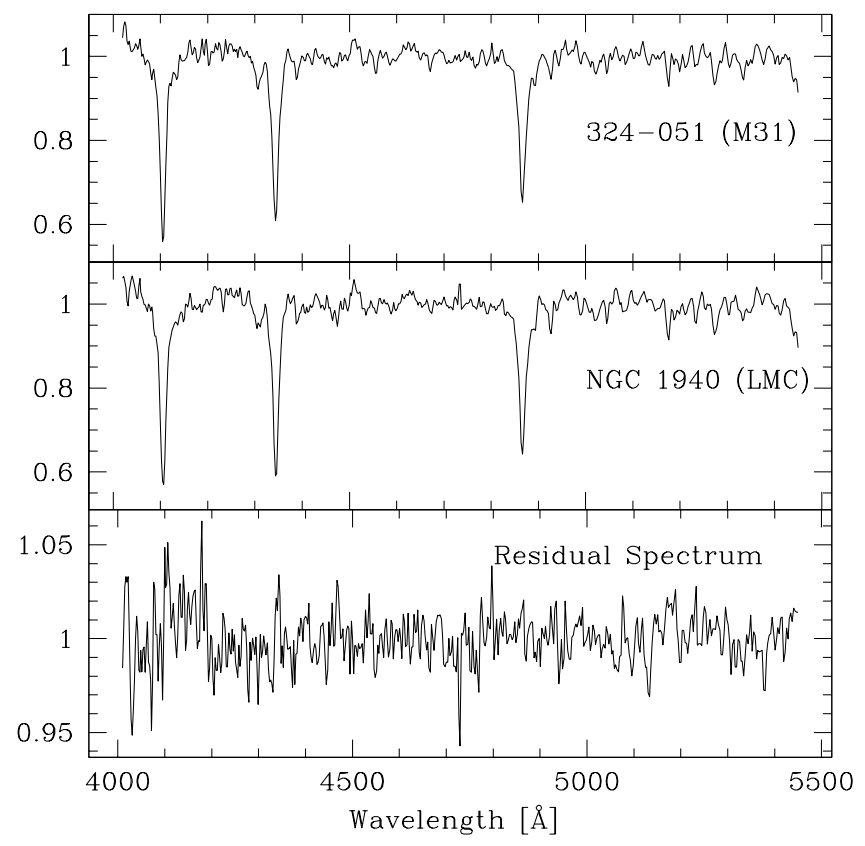

Fig. 10. - Comparison of the normalised spectra of 324-051 (Hubble V; top panel) and NGC 1940 (middle panel), which yielded the highest cross-correlation peak of all the LMC library (Beasley et al. 2002). The bottom panel shows the residual spectrum after dividing 324-051 by NGC 1940.

The next closest templates are NGC 2127 and NGC 2041 which are SWB III and II types respectively, corresponding to an age range of 30-200 Myr using the Bica et al. (1992) calibration. Girardi et al. (1995) obtained an age of 100 Myr for NGC 2041 based upon a calibration of the photometric $S$-parameter, slightly older than the Bica et al. calibration predicts. The work of Cohen (1982) suggests SWB III-types are closer to 500 Myr. This is an appreciable age-range, particularly when one considers that a 30 Myr cluster will be dominated by B stars, whereas in a 500 Myr stellar population A/F stars will be the dominant source of light. The spectrum of Hubble V (Figure 8) suggests a population of stars cooler than B, closer to late-A, due to the presence of metal-lines (particularly G-band) in the spectra, and the roll-over in the spectrum at $\sim 4000 \AA$. On the basis of the spectrum alone, the cluster is unlikely to be significantly younger than 100 Myr. Moreover, it should be noted that if the LMC templates are more metal-poor than the M31 clusters (a distinct possibility considering the mass-metallicity relation of galaxies), then due to age-metallicity degeneracy a younger, more metal-poor LMC template will yield the best correlation match with a slightly older, more metal-rich M31 cluster.

To conclude, we obtain an age-determination for Hubble V of 100-500 Myr, in good agreement with previous determinations, under the assumptions that the chemical composition of the young M31 clusters (metallicity, abundance ratios) are similar to those of the LMC system. 
We have performed the previous exercise for the remaining young M31 spectra, each time choosing the three closest cross-correlation templates to the cluster in question. Our results are summarized in Table 7. According to the LMC templates, the M31 clusters span a significant range of ages from between 10-30 Myr (327-053) up to 0.8-2 Gyr (292-010). The relative age rankings are qualitatively supported by the appearance of the cluster spectra in Figure 8.

We now appeal to the predictions of stellar population models for more information regarding these objects. To estimate the cluster ages and metallicities we have employed the evolutionary population synthesis models of Bruzual \& Charlot (2003; hereafter BC03). In the optical range which we are interested in (3670-6200 $⿱$ ) $)$, the BC03 models employ the recent stellar library (STELIB) of Le Borgne et al.(2003). The library, which consists of 249 stars taken at a resolution of $3 \AA$ (FWHM), covers with varying degrees of completeness the metallicity range $-2.0<[\mathrm{Fe} / \mathrm{H}]<$ +0.50 , luminosity classes I to V, and spectral types O5 to M9. Perhaps the most serious limitation of these (and other) models for our purposes is the lack of hot stars $\left(T_{\text {eff }}>10,000 \mathrm{~K}\right.$ ) at non-solar metallicities. This, however, should not be too problematic since we expect the abundances of the young M31 clusters to be at or near solar metallicity.

We show in Figure 11 the evolution of the Lick-defined $\mathrm{H} \beta$ index as a function of age and metallicity. The models shown adopt the Chabrier (2003) IMF, with a lower mass cut-off of $0.1 \mathrm{M}_{\odot}$ and upper mass cut-off of $100 \mathrm{M}_{\odot}$. The use of a Salpeter IMF has small effect on the optical indices in question here (BC03). According to the $\mathrm{BC} 03$ models, the $\mathrm{H} \beta$ index increased rapidly with time until $\sim 300 \mathrm{Myr}$, as turn-off A-stars come to dominate the integrated light. The strength of the index then decreases monotonically with age. In this regime, age is the single-most important influence on this index, whereas metallicity is a significant, but less important contributer. We have plotted our $\mathrm{H} \beta$ measurements for the eight young clusters in Figure 11, which range from 3.12 - $6.83 \AA$. In themselves, these suggest an age range between 0.02-5 Gyr. The higher-order Balmer lines show very similar behavior to that of $\mathrm{H} \beta$. 


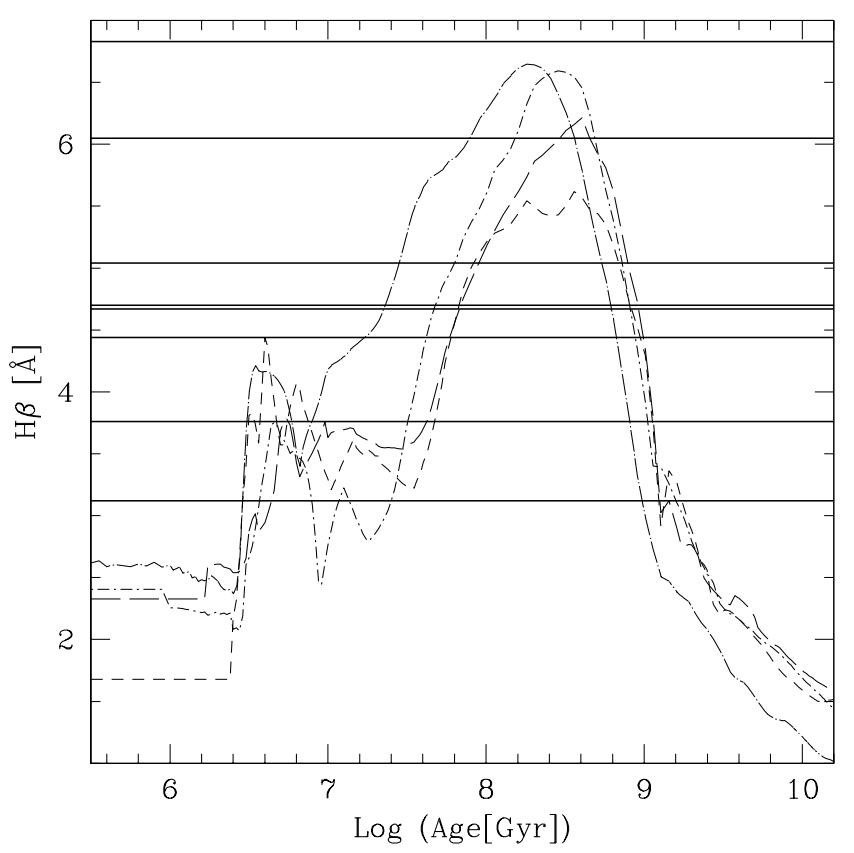

Fig. 11. - Evolution of the Lick $\mathrm{H} \beta$ index according to the Bruzual \& Charlot (2003) evolutionary population synthesis models, adopting the Chabrier (2003) IMF. Curves in different line-types represent metallicities $\mathrm{Z}_{\odot}([\mathrm{Fe} / \mathrm{H}]): 0.004(-0.64), 0.008(-0.33), 0.02(+0.09)$ and $0.05(+0.56)$ (short-dashed, long-dashed, dot-short dashed and dot-long dashed lines respectively). The thick horizontal lines are the $\mathrm{H} \beta$ measurements of the eight young clusters.

As has been noted before, the relatively narrow definition of the Lick $\mathrm{H} \beta$ index is not particularly well suited for very young stellar populations (e.g., Larsen \& Brodie 2002). However, we do not rely solely on the Balmer lines for age discrimination. Following the method outlined in Proctor et al. (2004), we have determined the ages and metallicities of the clusters by fitting all our measured Lick indices to the predictions of the BC03 models. Using the entire set of Lick indices maximizes the amount of age and metallicity information present in the spectra, since each index is individually sensitive to age and metallicity (and chemical abundance ratio variations; Proctor \& Sansom 2002). This approach is particularly useful in this case since in these young clusters the metal-lines are extremely diluted.

BC03 provide Lick indices for their model SEDs which cover the age-range $0.125 \mathrm{Myr}-20$ Gyr, and a metallicity range of $0.0001-0.05 \mathrm{Z}_{\odot}$. In this case, we use Lick indices measured off the BC03 SEDs, not those derived using the Lick fitting functions since our data is not corrected to the Lick system. As is the case for the Lick stellar library, the STELIB library is based on solar neighborhood stars. As such, it reflects the solar neighborhood abundance pattern. This creates an abundance ratio bias which is implicit in stellar population models which employ empirical stellar 
libraries (Trager et al. 2000; Thomas \& Maraston 2002; Proctor \& Sansom 2002). For very young systems correcting for this bias is highly uncertain, and therefore we make no correction to the BC03 models. This should not introduce serious errors in our derived abundances near to solar metallicity (Proctor et al. 2004).

For each cluster, we perform a $\chi^{2}$ fit of all our measured indices to each combination of age and metallicity in the BC03 models. The age and metallicity of the model which is best found to fit these data are then adopted as the best age and metallicity estimate of the particular cluster in question. For detailed discussions of the method applied to galaxy spectra see Proctor \& Sansom (2002) and Proctor et al. (2004). The results of our $\chi^{2}$ analysis are given in Table 8, and we show the 'age-metallicity' relation for these clusters in Figure 12. For comparison, we have also performed the same analysis for a subset of the Beasley et al. (2002) LMC clusters.

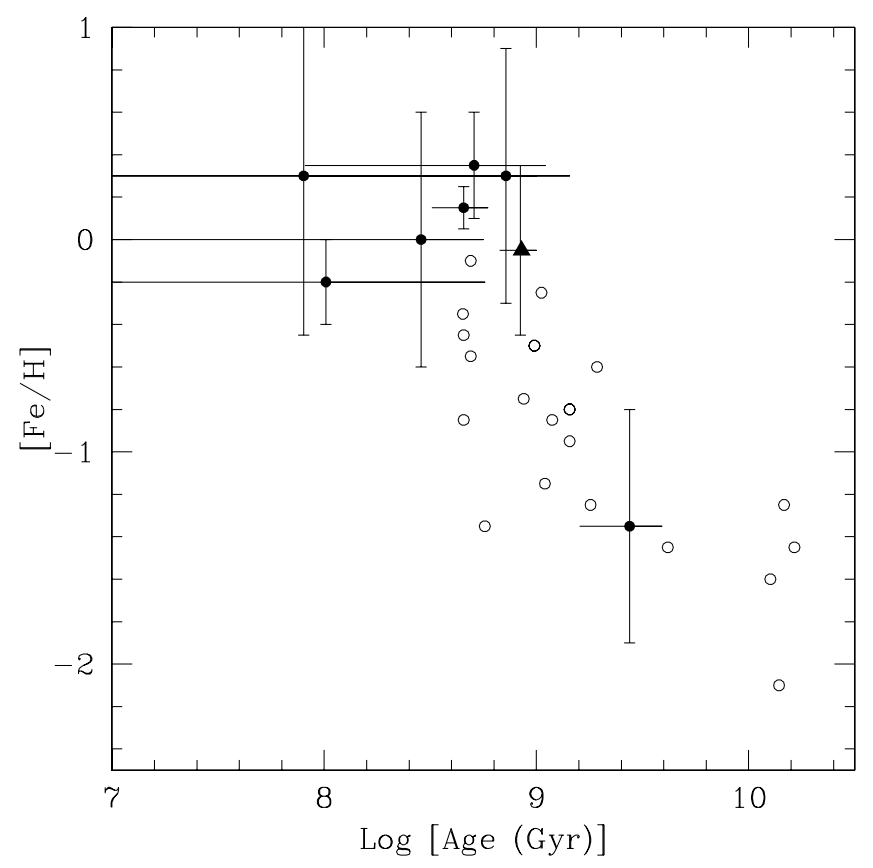

Fig. 12.- Ages and metallicities for the young M31 clusters (filled circles with error bars) and LMC clusters from Beasley et al. (2002; open circles, error bars omitted for clarity) based on the predictions of the Bruzual \& Charlot (2003) stellar population models. The position of Hubble V, the NGC 205 cluster, is marked with a solid triangle.

The majority of the ages derived from the BC03 models are consistent with those obtained from the LMC cluster templates. However, the BC03 models do generally predict older ages than these templates. This is consistent with the idea that the LMC templates are somewhat more metal-poor than the young M31 clusters. For Hubble V, we obtain an age of 0.51 \pm 0.59 Gyr, which is consistent with previous studies (Da Costa \& Mould 1988; Lee 1996). Figure 12 shows that, 
with the exception of 292-010, all of the young clusters are less than 1 Gyr old, and are very close to solar metallicity. Cluster 292-010 appears to be closer to 3 Gyr old, and is also somewhat more metal-poor. We conclude that our age determinations are in reasonable accord with the LMC templates, and henceforth we adopt the age and metallicity estimates for the young clusters based on the BC03 models which are given in Table 8. We are now in a position to estimate the masses of the clusters.

We have de-reddened the V-band apparent magnitudes of the clusters using the mean reddening, $\mathrm{E}(\mathrm{B}-\mathrm{V})=0.22$, derived by Barmby et al. (2000) and adopting $R_{V}=3.2$ (Cardelli, Clayton \& Mathis 1989). Assuming a distance modulus to M31 of $\mu=24.47$ (Holland 1998; Staneck \& Garnavich 1998), we obtain absolute magnitudes, and hence total V-band luminosities for the clusters. $M / L_{V}$ ratios for the clusters come from BC03, based upon our BC03 age determinations. This has the benefit of adding internal consistency to our calculations. In Table 8 we list the adopted $M / L_{V}$ ratios and estimated masses for the young M31 clusters. Estimating the uncertainties in our mass determinations is difficult, a major contribution to the error budget is the unknown reddening in the disk of M31. An increase in our adopted mean reddening by a factor of $2(\mathrm{E}(\mathrm{B}-\mathrm{V})=0.44)$ would roughly increase our mass estimate by the same factor. An adopted factor of 2 uncertainty in the values given in Table 8 is not unreasonable. The Chabrier (2003) IMF used in the BC03 models predicts that aging these clusters to $\sim 13$ Gyr will result in their fading by up to four magnitudes. Given their current absolute magnitude range of $-8.57 \leq M_{V} \leq-7.24$, this will result in clusters with $-4.57 \leq M_{V} \leq-3.24$, which is some four magnitudes fainter than the peak of the Galactic GC luminosity function.

A further consideration in this mass calculation is the effect of dynamical evolution, both internal and external to the clusters (e.g., Fall \& Zhang 2001). Several effects are expected to occur on different time-scales which will act to lower the cluster masses. For ages $\leq 10^{8}$ years, stellar winds and supernovae will remove some $\sim 30 \%$ of the initial mass from a cluster, whereas for clusters older than this, two-body relaxation, bulge and/or disk shocking and dynamical friction will all act upon the clusters to varying degrees. The survival of Galactic open clusters (OCs) at a given mass depends crucially upon their linear size (Wielen 1971). Massive, but small clusters, will feel the effects of dynamical relaxation most strongly, whereas more extended clusters will be more susceptible to the effects of the galaxian tidal field. For the M31 clusters in question, with masses $\sim 10^{4} \mathrm{M}_{\odot}$, we expect two-body relaxation to be the primary source of mass evolution (Gnedin \& Ostriker 1997). Obtaining structural parameters for these clusters will shed light on this important issue. Suffice to say that our mass estimates do not correct for any dynamical evolution.

The cluster masses and 'faded' absolute magnitudes we derive are relatively modest in GC terms. Some $90 \%$ of the Galactic GC system have masses $\geq 1 \times 10^{5} \mathrm{M}_{\odot}$. The only cluster in the sample that lies in this GC mass regime is the slightly older, more metal-poor 292-010, which lies out of the plane of the disk (see later). For reference, one of the least massive GCs known in the Galaxy is AM-4, which has an absolute magnitude, $M_{V}=-1.24$. Assuming $M / L_{V}=2$ for an old stellar population, we obtain a mass of $8.4 \times 10^{3} \mathrm{M}_{\odot}$ for this cluster. 
An important question here is whether these are actually young GCs, OCs, or lie somewhere else in the mass spectrum of star clusters. After Larsen \& Richtler (1999), in Figure 13 we plot the young M31 clusters in the $M_{V}$-age plane compared to the Galactic OC catalogue of Lyngå (1987), and the young, massive clusters (YMCs) identified in a sample of nearby spirals by Larsen \& Richtler (1999).

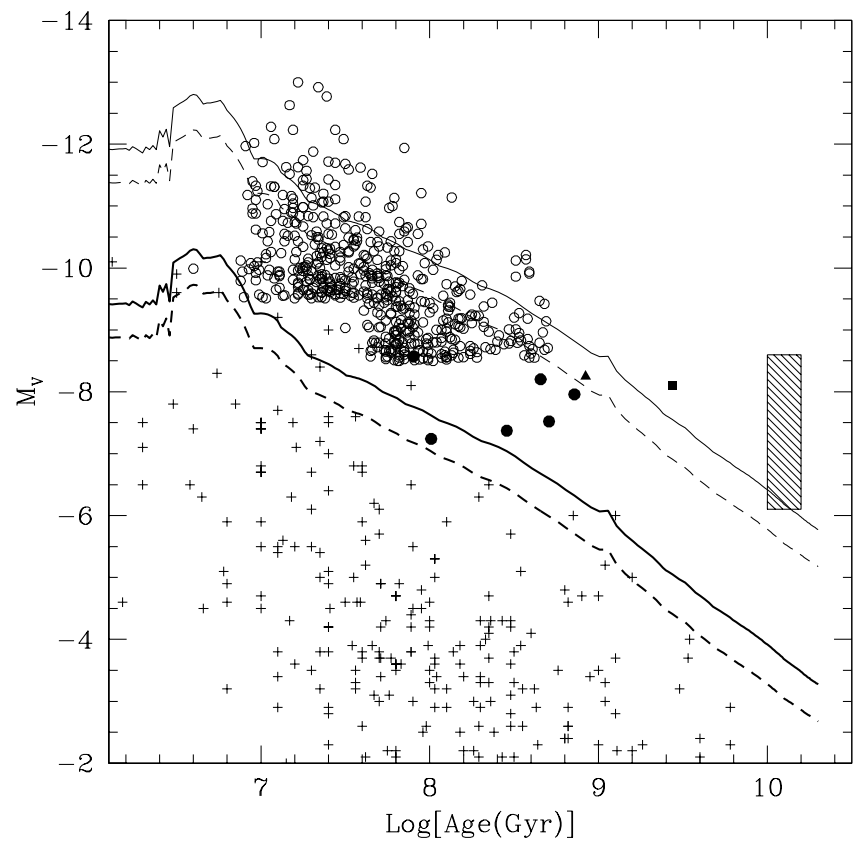

Fig. 13. - Comparison of the young M31 clusters (solid symbols), open cluster data from Lyngå (1987; plus signs) and young massive cluster data from Larsen \& Richtler (1999; open circles) in the $M_{V}$-log(age) plane. Hubble V in NGC 205 is marked as a triangle, 292-010 which lies out of the plane of the disk is marked as a square. The hatched region denotes the area occupied by Milky Way GCs with mean $M_{V}=-7.4$, and a dispersion of 1.2 mag. Lines represent the predictions of the Bruzual \& Charlot (2003) models for the fading with age of a cluster at masses $M=10^{4} \mathrm{M}_{\odot}$ (thick lines) and $M=10^{5} \mathrm{M}_{\odot}$ (faint lines) at solar metallicity. Dashed and solid lines represent Salpeter and Chabrier IMFs respectively.

The comparison between the young M31 clusters, the OCs and YMCs is not strictly fair for two reasons. Firstly, ages are derived for these latter two cluster types using integrated colors, whereas the M31 clusters have spectroscopically derived ages. Secondly, and perhaps more importantly, the selection functions of the three cluster types are very different. However, Figure 13 is revealing. The Galactic OCs from Lyngå (1987) are rather well bounded by the BC03 models for $M=10^{4} \mathrm{M}_{\odot}$ at solar metallicity. Very few are found to lie above this mass for the Chabrier IMF. With the exception of 327-053, all the M31 clusters are well separated from the upper envelope of the Galactic OCs. In fact, the M31 clusters roughly cover the mass range of Larsen \& Richtler's (1999) YMCs, although 
are somewhat older. This is probably not a real effect, but reflects a bias in the Larsen \& Richtler sample in that they preferentially obtained data for the bright end of the YMC luminosity function, which are intrinsically younger YMCs.

Also in Figure 13 we show the position of a GC at the turnover of the GCLF $\left(M_{V}=-7.4\right)$, with an observed dispersion of $1.2 \mathrm{mag}$. As the young M31 clusters fade and follow the locus of the models, they will occupy an area slighly below this region, between the GCs and OCs. Therefore, at present, we cannot say for certain whether we are seeing massive OCs, or relatively low-mass GCs. High-resolution imaging, and dynamical mass estimates will distinguish between these two possibilities.

With the exceptions of Hubble V (an NGC 205 cluster) and 292-010, all the clusters lie at or around solar metallicities, are $<1$ Gyr old, and are projected onto the disk of M31 (Figure 1). Cluster 292-010 lies $\sim 10 \mathrm{kpc}$ to the south-west of NGC 205, approximately $7 \mathrm{kpc}$ away from the disk. Are we, in fact, looking at a disk system of young clusters in M31? We first need to redetermine the velocities of these young clusters prior to looking at their kinematics, since their broad Balmer lines lead to serious systematic errors when their spectra are cross-correlated against classical $\mathrm{G}$ and $\mathrm{K}$ templates (Barmby et al., 2000). This possibility may afflict part of the Perrett et al. (2002) sample. We derive new velocities for the young clusters by cross-correlation with a series of A- and F- star templates from the STELIB spectral library. These new velocities and their respective uncertainties are given in Table 8. In Figure 14, we plot the rotation curve of the six young clusters compared to the rotation curve derived from HII regions of M31 by Rubin \& Ford (1970), and the HI curve of Kent (1989) 


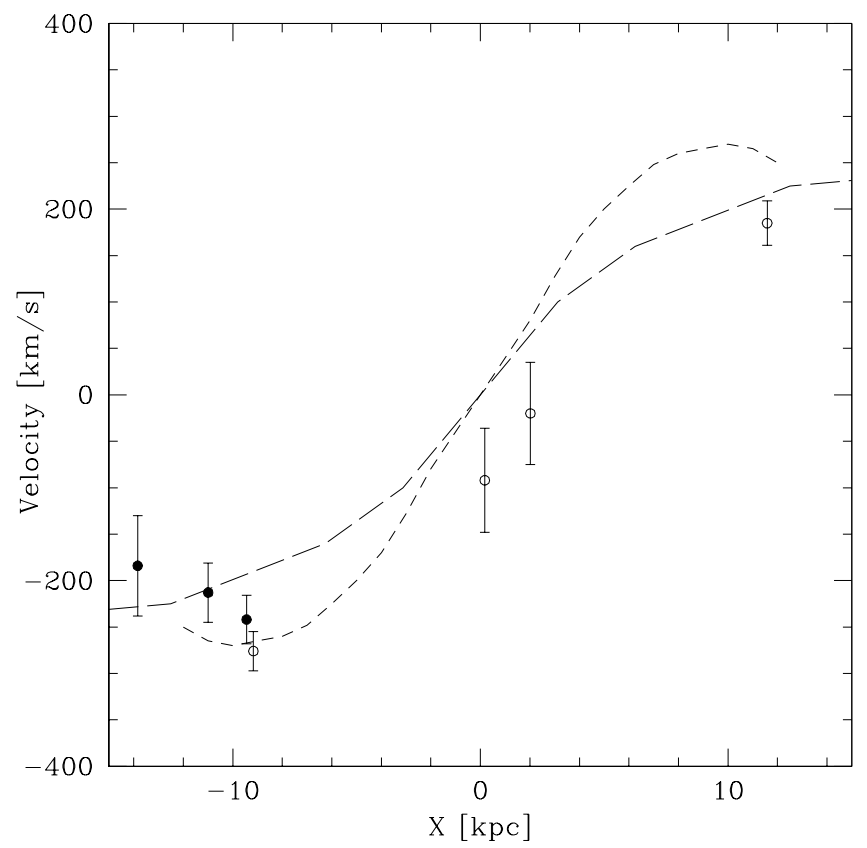

Fig. 14.- Velocity versus the projected distance along the major axis of M31. The six young clusters are compared to the rotation of M31 HII regions from Rubin \& Ford (1970; short-dashed line) and the HI rotation curve of Kent (1989; long-dashed line) assuming the same distance modulus. The HI and HII data have been folded on the $x$ - and $y$-axes, and extrapolated interior to $3 \mathrm{kpc}$. All velocities have been corrected to the M31 rest frame by $+300 \mathrm{~km} / \mathrm{s}$. Solid symbols represent the three clusters identified as possessing thin disk kinematics by Morrison et al. (2004). The young clusters show evidence of rotation similar to that of M31's gas disk.

The clusters show signs of rotating in the same sense as, and with a similar amplitude to, these HI and HII data. Unfortunately, the small sample and low-resolution of our spectra are unsuitable for a detailed analysis of this issue. Interestingly, on the basis of accurate velocities for 200 M31 GCs (Perrett et al. 2002), Morrison et al. (2004) have suggested the existence of a thin disk system of globular clusters in M31. This is in contrast to the Milky Way, which seems to have no such system. Moreover, these authors showed that, based upon the Perrett et al. metallicities, the clusters cover a metallicity range $-2.0 \leq[\mathrm{Fe} / \mathrm{H}] \leq 0$. This is significantly more metal-poor than the Milky Way stellar thin disk. Morrison et al. (2004) argued that, although they had no secure age estimates for these disk clusters, the fact that the disk clusters extend down to such low metallicities was indicative that the thin disk of M31 was likely to be old ( $\sim 10 \mathrm{Gyr})$.

Four of the six disk clusters in our sample are included in the Morrison et al. (2004) study, namely 314-037, 321-046, 327-053 and 380-313. Of these four, three have residuals from a disk model $\leq 0.75$, corresponding to thin-disk kinematics (see Morrison et al. 2004 for the precise definitions): 
314-037(0.57), 321-046(-0.05) and 327-053(0.25). These are consistent with their definition of belonging to a thin disk population, and indeed these clusters lie near the rotation curves shown in Figure 14. However, rather than being old, metal-poor disk clusters as suggested by Morrison et al.(2003), we find that these clusters are young and at approximately solar metallicity. We suggest that age-metallicity degeneracy, and the lack of Balmer lines in the Perrett et al. (2002) spectra,

lead these authors to conclude incorrectly these were metal-poor rather than young objects. A full census of the ages of these disk clusters is required to investigate this point.

\section{Discussion}

There appear to be at least two striking differences between the M31 GC system and that of the Milky Way.

\section{(i). Nitrogen Enhancement}

Since the work of Burstein et al. (1984) it has been suggested that CN in the integrated spectra of M31 GCs is enhanced relative to Milky Way GCs at the same metallicity. We have shown that this remains true for GCs in the range $-1.5 \leq[\mathrm{Fe} / \mathrm{H}] \leq-0.3$ (the metallicity range of the Milky Way GCs in our sample). Moreover, both the M31 and Milky Way GCs are enhanced in $\mathrm{CN}$ when compared to elliptical galaxies, which themselves show differences when divided into field and cluster samples (Sánchez-Blázquez et al. 2003). The very existence of CN variations suggest than $\mathrm{N}$ is the main culprit, since $\mathrm{C}$ is expected to be effectively absorbed into the $\mathrm{CO}$ molecule. This is supported by observations of the NH feature at $\lambda 3360 \AA$ (Ponder et al. 1998; Li \& Burstein 2004). The behaviour of $\mathrm{C}$ is less clear, although from the point of view of stellar evolution, $\mathrm{C}$ is expected to be destroyed in favor of $\mathrm{N}$ if the sum of $\mathrm{C}+\mathrm{N}+\mathrm{O}$ is preserved. A better understanding of the behavior of $\mathrm{C}$ and $\mathrm{O}$ in GCs may benefit from a comprehensive study of the $\mathrm{CO}$ feature at $2.3 \mu \mathrm{m}$ (e.g., Davidge 1990).

Varying CN enhancements in the integrated spectra of Galactic and M31 clusters is particularly interesting when one considers the $\mathrm{CN}$ variations known to exist between stars in individual Galactic globulars. The best documented case is that of 47 Tucanae. Norris \& Freeman (1979) found that the giants in this cluster exhibited CN-strong and CN-weak bimodality from the CN molecular band at $\lambda 4150 \AA$. This bimodality has since been observed to several magnitudes below the main sequence turn-off in Galactic GCs, along with the discovery that $\mathrm{CN}$ and $\mathrm{CH}$ are anti-correlated as expected from the CNO cycle (e.g., Cannon et al. 1998; Gratton et al. 2002; Briley, Cohen \& Stetson 2002, 2004; Harbeck, Smith \& Grebel 2003). Although no such studies exist for the much more distant M31 GCs, Tripicco (1989) and Tripico \& Bell (1992), showed that, on the basis of near-UV integrated spectroscopy and synthetic spectra, CN enhancement in the M31 GCs likely arises from CN-strong giants and $\mathrm{CN}$-strong dwarfs. Thus, the luminosity dependence of $\mathrm{CN}$ appears to exist in M31 GCs as well as Milky Way clusters.

As discussed comprehensively by Cannon et al. (1998), and summarized by Harbeck et al. 
(2003), three scenarios have been discussed to explain these puzzling chemical inhomogeneities; internal evolution of GC stars, GC self-pollution from evolved stars and primordial differences or GC self-enrichment.

Both the internal evolution and GC self-pollution scenarios have difficulty explaining the observed bimodality in CN in general, and its existence well below the main-sequence turn-off in GCs. $\mathrm{CNO}$ variations are expected to occur on the giant branch from standard mixing theory, where the convective envelope reaches it closest penetration to the CNO burning shell. However, this is not expected in stars well below the main-sequence turn-off, which are not expect to undergo deep mixing (Renzini \& Voli 1981). Self-pollution from asymptotic giant branch (AGB) stars are expected to eject CNO products through stellar winds, which may be deposited upon the rest of the stars in the cluster (Ventura et al. 2001). However, such accreted material is expected to be diluted in red giant branch (RGB) stars which undergo deep mixing. This is generally not observed, except possibly in a few metal-poor GCs (Suntzeff 1981; Carbon et al. 1982).

The primordial scenario argues that the proto-globular clouds had a range of chemical compositions, and a collision between two such clouds may result in the observed bimodality, if the gas remained unmixed prior to the onset of star formation. This picture is not unreasonable in an early proto-galactic environment, when the cross-section of cloud-cloud collisions is expected to be high (Bekki et al. 2004). However, this scenario faces the constraint in that the iron-peak elements show very little dispersion in individual GCs (e.g. Kraft 1994).

All of the M31 GCs in our sample have similar metallicities and luminosities to their Galactic counterparts, and lie on the same fundamental plane (Barmby, Holland \& Huchra 2002). These clusters are enhanced in CN with respect to the Milky Way GCs, which themselves show cluster to cluster variations (Cannon et al. 1998). It seems unlikely that internal-evolution or self-pollution would operate preferentially in the M31 GCs, but not in Milky Way clusters, particularly since the M31 GC dwarfs are likely CN-strong (Tripicco \& Bell 1992; Li \& Burstein 2004). Unless there are significant age differences between the Milky Way GCs and all the M31 clusters in our sample (which we have not observed, Paper II), it seems highly likely that the source of the CN enhancement was external to the M31 cluster system.

Although highly speculative, we are forced to conclude that the nitrogen enhancement in M31 GCs must be primordial in origin. The M31 proto-globular clouds were presumably enriched in nitrogen at some early epoch, whereas the proto-globular clouds of the Milky Way GCs were not. Much of the remaining nitrogen-rich material must have become incorporated into the bulk of the galaxy itself, although the M31 bulge shows significantly lower CN than the M31 GCs. With the advent of larger aperture telescopes, fine abundance analysis of individual halo stars in M31 would be extremely interesting in this regard.

\section{(ii). Young Disk Clusters}

Six of the young clusters in our sample appear to be disk clusters. They are projected on to the M31 disk, appear to rotate with the HI gas, and have approximately solar metallicities. 
Interestingly, one non-disk cluster appears to be somewhat older ( $\sim 3 \mathrm{Gyr})$, and lies approximately $7 \mathrm{kpc}$ away from the plane of the disk. On the basis of our mass estimates, it is presently not clear whether these objects are true GCs. Provided that they survive the dual effects of the tidal field of M31, and two-body relaxation, they will possibly evolve to populate the fainter end of the GCLF, or populate the bright end of the OCLF.

Are these clusters unusual? The work of Larsen \& Richtler (1999, 2000) highlighted the fact that young, massive clusters are actually a common feature of local spiral galaxies. The presence of young disk clusters in M31 is therefore not surprising. Based on a sample of 31 nearby spirals, Larsen \& Richtler (2000) found a correlation between the specific $U$-band YMC luminosity, $T_{L}(U)$, and the star formation rate (SFR) of the galaxies as traced by the far infrared luminosity of the host galaxy.

It is interesting to ask the question, where does M31 lie on this relation? Using equation (7) of Larsen \& Richtler (2000), based upon the far-infrared luminosity of M31, we obtain a normalised SFR for this galaxy of: $1.9 \times 10^{-4} \mathrm{M}_{\odot} y r^{-1} k p c^{-2}$. Assuming that all the metal-poor $([\mathrm{Fe} / \mathrm{H}]<-1.0)$ disk clusters in the Morrison et al. (2004) sample are young clusters, and adopting a mean $U$ band absolute magnitude of $\left\langle M_{U}\right\rangle=-6.7$ (Barmby et al. 2000), we obtain $T_{L}(U)=0.02$. Clearly the sample of M31 disk clusters is not complete, but these values suggest that M31 lies on the Larsen \& Richtler (2000) relation, and in the reasonably low SFR, low $T_{L}(U)$ regime (see figures $3 \& 5$ of Larsen \& Richtler 2000). This lends support to the idea that these objects are not open clusters in M31, and may represent the precursors of true thin disk globular clusters.

A system of young disk globular clusters is not seen in the Milky Way, and if M31 is truly forming disk GCs then this has important implications for galaxy formation theories. GCs are thought to trace the early episodes of the collapse of their host galaxy (e.g., Searle \& Zinn 1978; Forbes, Brodie \& Grillmair 1997) as well as later accretion (Coté, Marzke \& West 1998) and galaxy merging (Ashman \& Zepf 1992). Our results suggest that GCs may also trace the build up of galaxy disks, both at the present epoch and at higher redshifts. Indeed, the very existence of such disk clusters is expected on the basis that disks are where the majority of present-day star formation occurs. Recent efforts to simulate GC formation in the CDM paradigm also identify that a large fraction of GCs are expected to form in disk-like structures at high redshift (Beasley et al. 2002; Kravtsov \& Gnedin 2003).

Morrison et al. (2004) have recently argued for the existence of a thin disk in M31, based on GC kinematics, which extends down to $[\mathrm{Fe} / \mathrm{H}] \sim-2.0$. Such an old disk is perhaps not unexpected from a theoretical standpoint, if it can avoid disruptive merging in the dense, early universe (Baugh et al. 1996; Abadi et al. 2003). The observational constraints in this regard are not so clear, since the stellar thin disk of the Milky Way is the only such disk heretofore known to exist. However, in general, spiral stellar disks are thought to be metal-rich, if not relatively young (Bell \& de Jong 2002).

We have found that, rather than being old and metal-poor, the M31 disk clusters are in fact 
young and metal-rich. A larger census of these young disk clusters is required to see if they populate the entire low-metallicity sample of Morrison et al.(2004), however the implication is clear. Based upon our cluster data, the thin-disk identified by Morrison et al. (2004) may in fact be significantly younger than 10 Gyr (possibly as young as the oldest $\sim 1$ Gyr cluster), with a metallicity not too dissimilar to that of the Milky Way disk.

Brown et al. (2003) recently reported the presence of a significant intermediate-aged (6-8 Gyr) and metal-rich $([\mathrm{Fe} / \mathrm{H}]>-0.5)$ population of stars in a minor-axis halo field of M31, based on deep HST/ACS imaging. These authors suggested that M31 may have undergone a nearly equal-mass merger, 6-8 Gyr in the past. The presence of an old thin disk as identified by Morrison et al. (2004) effectively ruled out such a scenario, since any disk would be disrupted in such an event. With the identification that the thin disk of M31 may be as young as $\sim 1$ Gyr, the suggestion by Brown et al. (2003) becomes more tenable.

Based upon the evidence of enhanced N, and a disk population of possible GCs in M31, it appears then that not all GC systems of spiral galaxies are born equal. Both the initial chemical enrichment, and star formation (possibly merger history) of these two galaxies have differed significantly. Future work required includes a full census of the young star clusters in M31, secure mass determinations of these clusters, further near-UV and IR spectroscopy to understand the role of $\mathrm{N}$ and $\mathrm{C}$ in GCs respectively, and extensive spectoscopic and NUV imaging surveys of a greater number of local spiral galaxies.

We thank Javier Cenarro, Michael Pierce and Michael Rich for useful comments and suggestions on the paper, and Chris Flynn and Brad Gibson for early discussions regarding this work. We also thank P. Sánchez-Blázquez for supplying her thesis data ahead of full publication, Søren Larsen for prividing the YMC data. We are also very grateful to Judy Cohen and John Blakeslee for supplying the LRIS Galactic GC spectra in digital form. Funding support comes from NSF grant AST 0206139. The data presented herein were obtained at the W.M. Keck Observatory, which is operated as a scientific partnership among the California Institute of Technology, the University of California and the National Aeronautics and Space Administration. The Observatory was made possible by the generous financial support of the W.M. Keck Foundation. This research has made use of the NASA/IPAC Extragalactic Database (NED), which is operated by the Jet Propulsion Laboratory, Caltech, under contract with the National Aeronautics and Space Administration.

\section{REFERENCES}

Abadi, M. G., Navarro, J. F., Steinmetz, M., \& Eke, V. R., 2003, ApJ, 591, 499

Ashman, K. M. \& Zepf, S. E. 1992, ApJ, 384, 50

Barmby, P., Holland, S., \& Huchra, J. P. 2002, AJ, 123, 1937

Barmby, P. \& Huchra, J. P., 2001, AJ, 122, 2458 
Barmby, P., Huchra, J. P., Brodie, J. P., Forbes, D. A., Schroder, L. L., \& Grillmair, C. J., 2000, AJ, 119, 727

Battistini, P. L., Bonoli, F., Casavecchia, M., Ciotti, L., Federici, L., \& Fusi-Pecci, F., 1993, A\&A, 272,77

Battistini, P., Bonoli, F., Braccesi, A., Federici, L., Fusi Pecci, F., Marano, B., \& Borngen, F., 1987, A\&AS, 67, 447

Battistini, P., Bonoli, F., Braccesi, A., Fusi-Pecci, F., Malagnini, M. L., \& Marano, B., 1980, A\&AS, 42,357

Baugh, C. M., Cole, S., \& Frenk, C. S., 1996, MNRAS, 283, 1361

Beasley, M. A., Forbes, D. A., Brodie, J. P., \& Kissler-Patig, M., 2004, MNRAS, 347, 1150

Beasley, M. A., Baugh, C. M., Forbes, D. A., Sharples, R. M., \& Frenk, C. S. 2002, MNRAS, 333, 383

Beasley, M. A., Hoyle, F., \& Sharples, R. M., 2002, MNRAS, 336, 168

Beasley, M. A., Sharples, R. M., Bridges, T. J., Hanes, D. A., Zepf, S. E., Ashman, K. M., \& Geisler, D., 2000, MNRAS, 318, 1249

Bekki, K., Beasley, M. A., Forbes, D. A., \& Couch, W. J., 2004, ApJ, 602, 730

Bellazzini, M., Cacciari, C., Federici, L., Fusi Pecci, F., \& Rich, M., 2003, A\&A, 405, 867

Bell, E. F. \& de Jong, R. S., 2000, MNRAS, 312, 497

Bica, E., Claria, J. J., \& Dottori, H. 1992, AJ, 103, 1859

Brodie, J.P. and Huchra, J.P., 1990, ApJ 362, 503

Brodie, J.P. and Huchra, J.P., 1991, ApJ, 379, 157

Brook, C. B., Kawata, D., Gibson, B. K., \& Flynn, C. 2003, ApJ, 585, L125

Brown, T. M., Ferguson, H. C., Smith, E., Kimble, R. A., Sweigart, A. V., Renzini, A., Rich, R. M., \& VandenBerg, D. A. 2003, ApJ, 592, L17

Bruzual, G. \& Charlot, S. 2003, MNRAS, 344, 1000

Burstein, D., Faber,S.M., Gaskell,C.M. and Krumm, N., 1984, ApJ 287, 586

Cannon, R. D., Croke, B. F. W., Bell, R. A., Hesser, J. E., \& Stathakis, R. A., 1998, MNRAS,298, 601

Carbon, D. F., Romanishin, W., Langer, G. E., Butler, D., Kemper, E., Trefzger, C. F., Kraft, R. P., \& Suntzeff, N. B. 1982, ApJS, 49, 207

Cardelli, J. A., Clayton, G. C., \& Mathis, J. S. 1989, ApJ, 345, 245

Chabrier, G. 2003, PASP, 115, 763

Chandar, R., Whitmore, B., Lee, M-.G., 2004, ApJ, accepted

Chandar, R., Bianchi, L., \& Ford, H. C., 1999, ApJS, 122, 431 
Cohen, J. G., Briley, M. M., \& Stetson, P. B. 2002, AJ, 123, 2525

Cohen, J. G., Blakeslee, J. P., \& Ryzhov, A., 1998 (CBR98), ApJ, 496, 808

Cohen, J. G. 1982, ApJ, 258, 143

Côté, P., Marzke, R. O., West, M. J., \& Minniti, D., 2000, ApJ, 533, 869

Côté, P., Mateo, M., Sargent, W. L. W., \& Olszewski, E. W., 2000, ApJ, 537, L91

Côté, P., 1999, AJ, 118, 406

Crampton, D., Cowley, A. P., Schade, D., \& Chayer, P., 1985, ApJ, 288,494

Da Costa, G. S. \& Mould, J. R. 1988, ApJ, 334, 159

Davidge, T. J. 1990, ApJ, 351, L37

Elson, R. A. W., Fall, S. M., \& Freeman, K. C. 1987, ApJ, 323, 54

Fall, S. M. \& Zhang, Q. 2001, ApJ, 561, 751

Forbes, D.A., Strader, J., \& Brodie, J.P., 2004, AJ, 127, 3394

Forbes, D. A., Brodie, J. P., \& Larsen, S. S. 2001, ApJ, 556, L83

Freeman, K. \& Bland-Hawthorn, J. 2002, ARA\&A, 40, 487

Frenk, C. S. \& White, S. D. M. 1982, MNRAS, 198, 173

Geisler, D., Piatti, A. E., Bica, E., \& Clariá, J. J. 2003, MNRAS, 341, 771

Gnedin, O. Y. \& Ostriker, J. P. 1997, ApJ, 474, 223

González, J.J., 1993, Ph.D. Thesis, Univ. California, Santa Cruz, Lick Obs.

Gottesman, S. T., Hunter, J. H., \& Boonyasait, V. 2002, MNRAS, 337, 34

Goudfrooij, P., Strader, J., Brenneman, L., Kissler-Patig, M., Minniti, D., \& Edwin Huizinga, J. 2003, MNRAS, 343, 665

Harbeck, D., Smith, G. H., \& Grebel, E. K. 2003, AJ, 125, 197

Hodge, P. W. \& Wright, F. W. 1967, Washington: Smithsonian Press

Holland, S. 1998, AJ, 115, 1916

Holland, S., Fahlman, G. G., \& Richer, H. B. 1996, AJ, 112, 1035

Huchra, J.P., Brodie, J.P., Caldwell, N., Christian, C. \& Schommer, R. 1996, ApJS, 102, 29

Huchra, J. P., Brodie, J. P., \& Kent, S. M. 1991, ApJ, 370, 495

Jensen, J., Mould, J., \& Reid, N. 1988, ApJS, 67, 77

Kent, S. M. 1989, AJ, 97, 1614

Kim, S. C., Lee, M. G., Geisler, D., Sarajedini, A., Armandroff, T. E., \& da Costa, G. S. 2002, IAU Symposium, 207, 174

King, I. R. 1966, AJ, 71, 64 
Kraft, R. P. 1994, PASP, 106, 553

Kravtsov, A.V. \& Gnedin, O.Y., 2003, preprint (astro-ph/0305199)

Kuntschner, H., Ziegler, B. L., Sharples, R. M., Worthey, G., \& Fricke, K. J. 2002, A\&A, 395, 761

Larsen, S. S. \& Brodie, J. P. 2002, AJ, 123, 1488

Larsen, S. S. \& Richtler, T. 1999, A\&A, 345, 59

Lee, H., Yoon, S., \& Lee, Y. 2000, AJ, 120, 998

Lee, M. G. 1996, AJ, 112, 1438

Leonardi, A. J. \& Rose, J. A. 2003, AJ, 126, 1811

Li, Y. \& Burstein, D. 2003, ApJ, 598, L103

Lyngå G, 1987, Catalogue of open cluster data. Computer based catalogue available through CDS, Strasbourg, France, and through NASA Data Center, Grenbelt, Maryland, USA, 5th edition

Madore, B. F. \& Arp, H. C. 1982, PASP, 94, 40

Martin, N. F., Ibata, R. A., Bellazzini, M., Irwin, M. J., Lewis, G. F., \& Dehnen, W. 2004, MNRAS, 348,12

Minitti, D. 1995, AJ, 109, 1663

Morrison, H. L., Harding, P., Perrett, K., \& Hurley-Keller, D. 2004, ApJ, 603, 87

Norris, J., Cottrell, P. L., Freeman, K. C., \& Da Costa, G. S. 1981, ApJ, 244, 205

Norris, J. \& Freeman, K. C. 1979, ApJ, 230, L179

Oke, J.B. 1990, AJ, 99, 1621

Oke, J.B., J.G.Cohen, M.Carr, J.Cromer, A.Dingizian, F.H.Harris, S.Labrecque, R.Lucinio, W. Schaal, H.Epps \& J.Miller, 1995, PASP, 107, 307

Olsen, K.A.G., Miller, B.W., Suntzeff, N.B., Schommer, R.A., Bright, J., 2004, preprint (astro$\mathrm{ph} / 0401619)$

Olsen, K. A. G., Hodge, P. W., Mateo, M., Olszewski, E. W., Schommer, R. A., Suntzeff, N. B., \& Walker, A. R. 1998, MNRAS, 300, 665

Olszewski, E. W., Schommer, R. A., Suntzeff, N. B., \& Harris, H. C. 1991, AJ, 101, 515

Perrett, K. M., Bridges, T. J., Hanes, D. A., Irwin, M. J., Brodie, J. P., Carter, D., Huchra, J. P., \& Watson, F. G. 2002, AJ, 123, 2490

Piatti, A. E., Geisler, D., Bica, E., \& Clariá, J. J. 2003, MNRAS, 343, 851

Ponder, J. M., et al. 1998, AJ, 116, 2297

Puzia, T. H., Saglia, R. P., Kissler-Patig, M., Maraston, C., Greggio, L., Renzini, A., \& Ortolani, S. 2002, (P03) A\&A, 395, 45

Rabin, D. M. 1981, Ph.D. Thesis, California Inst. of Tech., Pasadena 
Renzini, A. \& Voli, M. 1981, A\&A, 94, 175

Rhode, K. L. 2003, Ph.D. Thesis, Yale Univ., New Haven

Rich, R. M., Reitzel, D. B., Guhathakurta, P., Gebhardt, K., \& Ho, L. C. 2004, AJ, 127, 2139

Rose, J. A. 1985, AJ, 90, 1927

Rosenberg, A., Saviane, I., Piotto, G., \& Aparicio, A. 1999, AJ, 118, 2306

Rubin, V. C. \& Ford, W. K. J. 1970, ApJ, 159, 379

Salaris, M. \& Weiss, A. 2002, A\&A, 388, 492

Sánchez-Blázquez, P., Gorgas, J., Cardiel, N., Cenarro, J., \& González, J. J. 2003, ApJ, 590, L91

Sargent, W. L. W., Kowal, C. T., Hartwick, F. D. A., \& van den Bergh, S. 1977, AJ, 82, 947

Schaller, G., Schaerer, D., Meynet, G., \& Maeder, A. 1992, A\&AS, 96, 269

Schroder, L. L., Brodie, J. P., Kissler-Patig, M., Huchra, J. P., \& Phillips, A. C. 2002, AJ, 123, 2473

Searle, L., Wilkinson, A., \& Bagnuolo, W. G. 1980, (SWB) ApJ, 239, 803

Searle, L. \& Zinn, R. 1978, ApJ, 225, 357

Spinrad, H. \& Schweizer, F. 1972, ApJ, 171, 403

Stanek, K. Z. \& Garnavich, P. M. 1998, ApJ, 503, L131

Strader, J., Brodie, J.P., \& Forbes, D.A., 2004, AJ, 127, 3431

Suntzeff, N. B. 1981, ApJS, 47, 1

Thomas, D., Maraston, C., \& Bender, R. 2003, MNRAS, 339, 897

Trager, S. C. 2004, Origin and Evolution of the Elements, in Carnegie Observatories Centennial Symposia, Cambridge University Press, Eds A. McWilliam and M. Rauch, p391

Trager, S. C., Worthey, G., Faber, S. M., Burstein, D., \& Gonzalez, J. J. 1998, ApJS, 116, 1

Tripicco, M. J. \& Bell, R. A. 1995, AJ, 110, 3035

Tripicco, M. J. \& Bell, R. A. 1992, AJ, 103, 1285

Tripicco, M. J. 1989, AJ, 97, 735

van den Bergh, S. 1975, ARA\&A, 13, 217

van den Bergh, S. 1969, ApJS, 19, 145

Vazdekis, A. 1999, ApJ, 513, 224

Ventura, P., D'Antona, F., Mazzitelli, I., \& Gratton, R. 2001, ApJ, 550, L65

Wielen, R. 1971, A\&A, 13, 309

Worthey, G. \& Ottaviani, D.L., 1997, ApJS, 111, 445

Worthey, G. 1994, ApJS, 95, 107 
Worthey, G., Faber, S. M., Gonzalez, J. J., \& Burstein, D. 1994, ApJS, 94, 687

Zinn, R. and West, M. 1984, ApJS, 55,45

Zinn, R. 1985, ApJ, 293, 424

This preprint was prepared with the AAS $\mathrm{LAT}_{\mathrm{E}} \mathrm{X}$ macros v5.2. 
Table 1. Basic data for M31 globular clusters.

\begin{tabular}{|c|c|c|c|c|c|c|c|c|}
\hline $\mathrm{Name}^{a}$ & $\begin{array}{c}\mathrm{RA}^{b} \\
(\mathrm{~J} 2000)\end{array}$ & $\begin{array}{c}\text { Dec }^{b} \\
(\mathrm{~J} 2000)\end{array}$ & $\begin{array}{c}\mathrm{X}^{b} \\
(\operatorname{arcmin})\end{array}$ & $\begin{array}{c}\mathrm{Y}^{b} \\
(\operatorname{arcmin})\end{array}$ & $\begin{array}{c}\mathrm{Z}^{b} \\
(\operatorname{arcmin})\end{array}$ & $\begin{array}{l}\text { Velocity }^{b} \\
\left(\mathrm{kms}^{-1}\right)\end{array}$ & $\begin{array}{c}\mathrm{S} / \mathrm{N}^{c} \\
\left(\text { pixel }^{-1}\right)\end{array}$ & Notes \\
\hline $126-184$ & 04243.78 & 411242.98 & -2.8 & -2.0 & 3.5 & $-182 \pm 14$ & 73 & \\
\hline $134-190$ & 04251.72 & 411402.88 & -0.8 & -2.4 & 2.5 & $-401 \pm 32$ & 70 & \\
\hline $158-213$ & 04314.47 & 410720.58 & -3.4 & -9.9 & 10.5 & $-187 \pm 6^{d}$ & 120 & velocity standard \\
\hline $163-217$ & 04317.71 & 412745.53 & 13.0 & 2.2 & 13.2 & $-161 \pm 4^{d}$ & 170 & velocity standard \\
\hline $222-277$ & 04425.51 & 411412.62 & 10.2 & -16.2 & 19.1 & $-282 \pm 20$ & 30 & F-type spectrum, peculiar \\
\hline $225-280$ & 04429.78 & 412136.57 & 16.5 & -12.2 & 20.5 & $-167 \pm 8^{d}$ & 169 & velocity standard \\
\hline $234-290$ & 04446.50 & 412918.34 & 24.5 & -9.9 & 26.4 & $-234 \pm 16$ & 71 & \\
\hline $292-010$ & 03616.69 & 405826.64 & -58.5 & 47.1 & 75.1 & $-331 \pm 23$ & 32 & late $\mathrm{F}$ spectrum \\
\hline $301-022$ & 03821.61 & 400337.22 & -87.8 & -5.0 & 88.0 & $-30+20$ & 31 & \\
\hline $304-028$ & 03856.82 & $41 \quad 1028.81$ & -30.7 & 30.3 & 43.1 & $-353+15$ & 34 & \\
\hline $310-032$ & 03925.82 & 412333.46 & -17.0 & 34.0 & 38.0 & $-189 \pm 18$ & 35 & \\
\hline $313-036$ & 03944.52 & 405256.24 & -39.1 & 12.5 & 41.1 & $-446 \pm 6$ & 52 & \\
\hline $314-037$ & 03944.60 & 401408.23 & -69.9 & -11.2 & 70.7 & $-318 \pm 66$ & 17 & F0 spectrum \\
\hline $321-046$ & 04015.30 & 402746.86 & -55.5 & -7.5 & 56.0 & $-527 \pm 35$ & 30 & A-type spectrum \\
\hline $322-049$ & 04017.28 & 403905.83 & -46.3 & -0.8 & 46.3 & $-581 \pm 29$ & 40 & A-type spectrum \\
\hline $324-051$ & 04020.75 & 414050.79 & 3.0 & 36.4 & 36.5 & $-299 \pm 35$ & 69 & F-type spectrum, Hubble V \\
\hline $327-053$ & 04024.09 & 403622.75 & -47.7 & -3.5 & 47.8 & $-658 \pm 29$ & 71 & A-F spectrum \\
\hline $328-054$ & 04024.86 & 414023.74 & 3.1 & 35.5 & 35.6 & $-243 \pm 23$ & 30 & \\
\hline $337-068$ & 04048.45 & 421210.45 & 30.9 & 51.5 & 60.0 & $50 \pm 12$ & 178 & $\mathrm{CN}$ weak, $\mathrm{CH}$ strong? \\
\hline $347-154$ & 04222.95 & 415428.25 & 27.7 & 26.7 & 38.5 & $-312 \pm 36$ & 61 & \\
\hline $350-162$ & 04228.43 & 402452.18 & -42.2 & -29.2 & 51.4 & $-457 \pm 18$ & 39 & \\
\hline $365-284$ & 04436.77 & 421720.47 & 61.0 & 21.4 & 64.7 & $-78 \pm 13$ & 60 & \\
\hline $380-313$ & 04606.34 & 420053.23 & 58.5 & -1.9 & 58.5 & $-121 \pm 31$ & 43 & F0 spectrum \\
\hline $383-318$ & 04611.89 & $\begin{array}{lll}41 & 19 & 42.15\end{array}$ & 27.0 & -28.4 & 39.1 & $-250 \pm 10$ & 85 & \\
\hline 393-330 & 04701.22 & 412406.45 & 36.2 & -32.8 & 48.9 & $-331 \pm 10$ & 43 & \\
\hline $398-341$ & 04757.83 & 414844.63 & 62.0 & -25.6 & 67.1 & $-227 \pm 5$ & 44 & \\
\hline $401-344$ & 04808.52 & 414042.47 & 57.0 & -32.2 & 65.5 & $-273 \pm 52$ & 54 & \\
\hline NB16 & 04233.18 & 412017.12 & 2.0 & 4.2 & 4.6 & $-115 \pm 15$ & 142 & \\
\hline NB67 & 04234.18 & 411947.10 & 1.7 & 3.7 & 4.1 & $-113 \pm 17$ & 91 & \\
\hline NB89 & 04244.69 & 411441.97 & -1.1 & -1.0 & 1.5 & $-332 \pm 6$ & 110 & \\
\hline
\end{tabular}




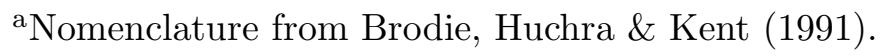

${ }^{\mathrm{b}}$ From Barmby et al. (2000).

${ }^{\mathrm{c}}$ Derived signal-to-noise measured at $5000 \AA$.

${ }^{\mathrm{d}}$ Echelle velocities from Peterson (2000)

Note. - X,Y,Z are in arcmins and defined in Huchra, Brodie \& Kent (1991) 
Table 2. Lick/IDS indices and uncertainties measured for the Galactic GCs in the Cohen et al.(1998) sample

\begin{tabular}{|c|c|c|c|c|c|c|c|c|c|c|c|c|c|c|}
\hline "Name & 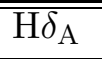 & $\overline{\overline{\mathrm{H}} \delta_{\mathrm{F}}}$ & 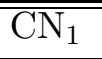 & $\overline{\mathrm{CN}_{2}}$ & Ca4227 & 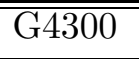 & 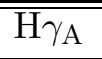 & 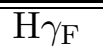 & Fe4384 & Ca4455 & 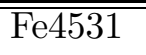 & $\mathrm{C}_{2} 4668$ & 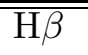 & Fe5015 \\
\hline NGC 6205 & $\cdots$ & $\cdots$ & $\cdots$ & $\ldots$ & 0.32 & 2.14 & 1.54 & 1.90 & 0.86 & 0.11 & 1.10 & 0.19 & 2.47 & 1.43 \\
\hline & $\cdots$ & $\cdots$ & $\cdots$ & $\cdots$ & 0.03 & 0.57 & 0.54 & 0.17 & 0.34 & 0.05 & 0.07 & 0.46 & 0.13 & 0.23 \\
\hline NGC 6121 & ... & $\ldots$ & ... & ... & 0.40 & 2.72 & 1.58 & 2.23 & 1.76 & 0.22 & 1.48 & 0.62 & 2.91 & 2.26 \\
\hline NCC 6838 & $\cdots$ & & & & 0.05 & 0.58 & 0.55 & 0.18 & 0.35 & 0.07 & 0.10 & 0.47 & 0.13 & 0.24 \\
\hline NGC 6838 & $\ldots$ & -0.34 & 0.005 & 0.023 & 0.81 & 5.30 & -4.57 & -1.11 & 3.13 & 0.57 & 2.55 & 2.10 & 1.43 & 3.79 \\
\hline & $\ldots$ & 0.12 & 0.003 & 0.003 & 0.03 & 0.57 & 0.54 & 0.17 & 0.34 & 0.04 & 0.06 & 0.46 & 0.13 & 0.22 \\
\hline NGC 6341 & $\ldots$ & $\ldots$ & $\ldots$ & $\ldots$ & 0.17 & 1.04 & 2.78 & 2.44 & 0.61 & 0.09 & 0.57 & 0.08 & 2.55 & 0.66 \\
\hline 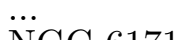 & ... & $\ldots$ & $\cdots$ & $\ldots$ & 0.03 & 0.57 & 0.54 & 0.17 & 0.34 & 0.04 & 0.07 & 0.46 & 0.13 & 0.23 \\
\hline NGC 6171 & $\cdots$ & $\cdots$ & $\cdots$ & $\ldots$ & $\ldots$ & -0.47 & 4.96 & 2.52 & 1.48 & 0.14 & -0.51 & 0.32 & 1.60 & 2.53 \\
\hline NGC 6356 & $\cdots$ & 060 & 0044 & 0067 & 075 & 0.61 & $\begin{array}{l}0.57 \\
-381\end{array}$ & 0.21 & 0.46 & 0.16 & 0.23 & 0.54 & 0.16 & 0.30 \\
\hline NGE 0000 & $\cdots$ & 0.13 & 0.003 & 0.003 & 0.04 & $\begin{array}{l}0.57 \\
0.57\end{array}$ & $\begin{array}{r}-.0 .01 \\
0.55\end{array}$ & $\begin{array}{r}-0.60 \\
0.18\end{array}$ & $\begin{array}{l}3.25 \\
0.34\end{array}$ & $\begin{array}{l}0.69 \\
0.05\end{array}$ & $\begin{array}{l}2.62 \\
0.08\end{array}$ & $\begin{array}{l}2.06 \\
0.46\end{array}$ & $\begin{array}{l}1.63 \\
0.13\end{array}$ & $\begin{array}{l}3.57 \\
0.23\end{array}$ \\
\hline NGC 6440 & $\ldots$ & $\ldots$ & $\ldots$ & $\ldots$ & $\ldots$ & $\ldots$ & $\ldots$ & $\ldots$ & $\ldots$ & $\ldots$ & $\begin{array}{l}0.00 \\
\ldots\end{array}$ & 2.42 & 1.44 & 3.90 \\
\hline & $\cdots$ & $\cdots$ & $\cdots$ & $\cdots$ & $\cdots$ & $\cdots$ & $\cdots$ & $\cdots$ & $\cdots$ & $\cdots$ & $\cdots$ & 0.46 & 0.13 & 0.23 \\
\hline NGC 6528 & $\cdots$ & $\cdots$ & $\cdots$ & ... & $\cdots$ & $\cdots$ & $\cdots$ & $\cdots$ & $\cdots$ & $\cdots$ & $\cdots$ & 3.88 & 1.83 & 5.00 \\
\hline $\operatorname{NCC} 6520$ & $\cdots$ & $\cdots$ & $\cdots$ & $\cdots$ & $\cdots$ & $\cdots$ & $\cdots$ & $\cdots$ & $\cdots$ & $\cdots$ & $\cdots$ & 0.47 & 0.13 & 0.23 \\
\hline NGC 6539 & $\cdots$ & $\cdots$ & $\cdots$ & $\cdots$ & $\cdots$ & $\cdots$ & $\cdots$ & $\cdots$ & $\cdots$ & $\cdots$ & $\cdots$ & 2.68 & 1.40 & 3.94 \\
\hline NGC 6553 & $\cdots$ & $\cdots$ & $\cdots$ & $\cdots$ & $\cdots$ & $\cdots$ & $\cdots$ & $\cdots$ & $\cdots$ & $\cdots$ & $\cdots$ & 0.51 & 0.15 & $\theta .27$ \\
\hline Nu & $\cdots$ & $\cdots$ & $\cdots$ & $\cdots$ & $\cdots$ & $\cdots$ & $\cdots$ & $\cdots$ & $\cdots$ & $\cdots$ & $\cdots$ & $\begin{array}{l}3.92 \\
0.46\end{array}$ & $\begin{array}{l}1.68 \\
012\end{array}$ & 5.08 \\
\hline N̈GC 6624 & $\begin{array}{l}\cdots \\
\ldots\end{array}$ & $\cdots$ & $\cdots$ & $\cdots$ & $\cdots$ & $\cdots$ & $\cdots$ & $\cdots$ & $\cdots$ & $\cdots$ & $\cdots$ & $\begin{array}{l}0.40 \\
183\end{array}$ & 171 & $\begin{array}{l}0.22 \\
3.61\end{array}$ \\
\hline & & $\ldots$ & $\ldots$ & $\ldots$ & $x_{-1}+2$ & $x_{-1}+2$ & $\ldots$ & $\ldots$ & $\cdots$ & $\ldots$ & $\cdots$ & 0.45 & 0.13 & 0.22 \\
\hline NGC 6760 & $\ldots$ & $\ldots$ & $\ldots$ & $\ldots$ & $\ldots$ & $\ldots$ & $\ldots$ & $\ldots$ & $\ldots$ & $\ldots$ & $\ldots$ & 2.15 & 1.38 & 4.11 \\
\hline & $\ldots$ & $\ldots$ & $\ldots$ & $\ldots$ & $\ldots$ & $\ldots$ & $\ldots$ & $\ldots$ & $\ldots$ & $\ldots$ & $\ldots$ & 0.47 & 0.13 & 0.23 \\
\hline
\end{tabular}


Table 2A. Lick/IDS indices and uncertainties measured for the Galactic GCs in the Cohen et al.(1998) sample.

\begin{tabular}{|c|c|c|c|c|c|c|c|c|c|c|}
\hline Name & $\begin{array}{l}\mathrm{Mg}_{1} \\
{[\mathrm{mag}]}\end{array}$ & $\begin{array}{c}\mathrm{Mg}_{2} \\
{[\mathrm{mag}]}\end{array}$ & $\begin{array}{c}\mathrm{Mg} b \\
{[\AA]}\end{array}$ & $\begin{array}{c}\text { Fe5270 } \\
{[\AA]}\end{array}$ & $\begin{array}{c}\text { Fe5335 } \\
{[\AA]}\end{array}$ & $\begin{array}{c}\text { Fe } 5406 \\
{[\AA]}\end{array}$ & $\begin{array}{c}\text { Fe } 5709 \\
{[\AA]}\end{array}$ & $\begin{array}{c}\text { Fe5782 } \\
{[\AA]}\end{array}$ & $\begin{array}{c}\mathrm{Na} \mathrm{D} \\
{[\AA]}\end{array}$ & $\begin{array}{l}\mathrm{TiO}_{1} \\
{[\mathrm{mag}]}\end{array}$ \\
\hline \multirow[t]{2}{*}{ NGC 6205} & 0.008 & 0.048 & 0.83 & 0.87 & 0.74 & 0.38 & 0.23 & 0.11 & 0.91 & 0.003 \\
\hline & 0.014 & 0.010 & 0.08 & 0.14 & 0.13 & 0.17 & 0.11 & 0.13 & 0.10 & 0.012 \\
\hline \multirow{2}{*}{ NGC 6121} & 0.031 & 0.094 & 1.57 & 1.26 & 0.96 & 0.51 & 0.47 & 0.36 & 1.68 & 0.009 \\
\hline & 0.014 & 0.010 & 0.08 & 0.14 & 0.13 & 0.17 & 0.11 & 0.13 & 0.10 & 0.012 \\
\hline \multirow[t]{2}{*}{ NGC 6838} & 0.074 & 0.164 & 2.71 & 2.17 & 1.95 & 1.32 & 0.78 & 0.57 & 2.28 & 0.023 \\
\hline & 0.014 & 0.010 & 0.08 & 0.13 & 0.13 & 0.17 & 0.11 & 0.13 & 0.10 & 0.012 \\
\hline \multirow[t]{2}{*}{ NGC 6341} & 0.004 & 0.029 & 0.52 & 0.48 & 0.43 & 0.18 & 0.09 & 0.04 & 0.68 & 0.003 \\
\hline & 0.014 & 0.010 & 0.08 & 0.13 & 0.13 & 0.17 & 0.11 & 0.13 & 0.10 & 0.012 \\
\hline \multirow[t]{2}{*}{ NGC 6171} & 0.041 & 0.118 & 1.81 & 1.50 & 1.21 & 0.68 & 0.49 & 0.47 & 1.86 & 0.017 \\
\hline & 0.014 & 0.010 & 0.12 & 0.17 & 0.17 & 0.19 & 0.12 & 0.14 & 0.12 & 0.012 \\
\hline \multirow{2}{*}{ NGC 6356} & 0.072 & 0.172 & 3.02 & 2.01 & 1.84 & 1.21 & 0.79 & 0.65 & 3.05 & 0.030 \\
\hline & 0.014 & 0.010 & 0.08 & 0.14 & 0.13 & 0.17 & 0.11 & 0.13 & 0.10 & 0.012 \\
\hline \multirow[t]{2}{*}{ NGC 6440} & 0.102 & 0.225 & 3.25 & 2.32 & 1.73 & 1.19 & 0.90 & 1.02 & 4.21 & 0.030 \\
\hline & 0.014 & 0.010 & 0.08 & 0.13 & 0.13 & 0.17 & 0.11 & 0.12 & 0.10 & 0.012 \\
\hline \multirow[t]{2}{*}{ NGC 6528} & 0.097 & 0.246 & 3.81 & 2.79 & 2.21 & 1.62 & 1.05 & 1.00 & 5.12 & 0.044 \\
\hline & 0.014 & 0.010 & 0.08 & 0.14 & 0.13 & 0.17 & 0.11 & 0.13 & 0.10 & 0.012 \\
\hline \multirow{2}{*}{ NGC 6539} & 0.084 & 0.193 & 2.81 & 2.14 & 1.54 & 1.19 & 0.92 & 0.96 & 4.05 & 0.037 \\
\hline & 0.014 & 0.010 & 0.10 & 0.15 & 0.15 & 0.18 & 0.11 & 0.13 & 0.11 & 0.012 \\
\hline \multirow{2}{*}{ NGC 6553} & 0.106 & 0.262 & 3.99 & 2.96 & 2.36 & 1.67 & 0.97 & 1.09 & 3.70 & 0.048 \\
\hline & 0.014 & 0.010 & 0.08 & 0.13 & 0.13 & 0.17 & 0.11 & 0.12 & 0.10 & 0.012 \\
\hline \multirow{2}{*}{ NGC 6624} & 0.048 & 0.161 & 2.83 & 1.93 & 1.59 & 1.07 & 0.65 & 0.57 & 2.47 & 0.031 \\
\hline & 0.014 & 0.010 & 0.07 & 0.13 & 0.12 & 0.17 & 0.10 & 0.12 & 0.10 & 0.012 \\
\hline \multirow{2}{*}{$\begin{array}{l}\text { NGC } 6760 \\
\ldots\end{array}$} & 0.095 & 0.225 & 3.30 & 2.30 & 1.78 & 1.29 & 0.83 & 0.87 & 3.51 & 0.045 \\
\hline & 0.014 & 0.010 & 0.08 & 0.14 & 0.13 & 0.17 & 0.11 & 0.13 & 0.10 & 0.012 \\
\hline
\end{tabular}


Table 3. Mean offsets and standard deviations $\left(\sigma_{\mathrm{rms}}\right)$ between P02 and CBR98 Indices at the Lick/IDS resolution, but uncalibrated to the Lick system.

\begin{tabular}{lcc}
\hline \hline Index & P02-CBR98 & $\sigma_{\text {rms }}$ \\
& & \\
\hline $\mathrm{H} \delta_{\mathrm{A}}$ & $\ldots$ & $\ldots$ \\
$\mathrm{H} \delta_{\mathrm{F}}$ & 0.1144 & $0.1144^{a}$ \\
$\mathrm{CN}_{1}$ & 0.0027 & $0.0027^{a}$ \\
$\mathrm{CN}_{2}$ & 0.0018 & $0.0018^{a}$ \\
$\mathrm{Ca} 4227$ & 0.0044 & $0.0044^{a}$ \\
$\mathrm{G} 4300$ & -0.5746 & $0.5746^{a}$ \\
$\mathrm{H} \gamma_{\mathrm{A}}$ & -0.5411 & $0.5411^{a}$ \\
$\mathrm{H} \gamma_{\mathrm{F}}$ & -0.1702 & $0.1702^{a}$ \\
$\mathrm{Fe} 4383$ & -0.3277 & $0.3277^{a}$ \\
$\mathrm{Ca} 4455$ & 0.0042 & $0.0042^{a}$ \\
$\mathrm{Fe} 4531$ & 0.0299 & $0.0299^{a}$ \\
$\mathrm{C}_{2} 4668$ & 0.5646 & 0.4533 \\
$\mathrm{H} \beta$ & -0.0027 & 0.1257 \\
$\mathrm{Fe} 5015$ & -0.8856 & 0.2142 \\
$\mathrm{Mg}{ }_{1}$ & -0.0087 & 0.0136 \\
$\mathrm{Mg} g_{2}$ & -0.0043 & 0.0096 \\
$\mathrm{Mg} b$ & -0.1616 & 0.0723 \\
$\mathrm{Fe} 5270$ & 0.0650 & 0.1309 \\
$\mathrm{Fe} 5335$ & 0.0025 & 0.1199 \\
$\mathrm{Fe} 5406$ & -0.1590 & 0.1689 \\
$\mathrm{Fe} 5709$ & 0.3026 & 0.1036 \\
$\mathrm{Fe} 5782$ & 0.0063 & 0.1235 \\
$\mathrm{NaD}_{\mathrm{TiO}}$ & -0.1975 & 0.0979 \\
$\mathrm{TiO}$ & 0.0076 & 0.0115 \\
& 0.0228 & 0.0123 \\
\hline & &
\end{tabular}

${ }^{\mathrm{a}} \sigma_{\text {rms }}$ taken to be the absolute difference. 
Table 4. Lick/IDS indices and uncertainties for M31 globular clusters.

\begin{tabular}{|c|c|c|c|c|c|c|c|c|c|c|c|c|c|c|}
\hline Name & $\begin{array}{c}\mathrm{H} \delta_{\mathrm{A}} \\
{[\AA]}\end{array}$ & $\begin{array}{c}\mathrm{H} \delta_{\mathrm{F}} \\
{[\AA]}\end{array}$ & $\begin{array}{c}\mathrm{CN}_{1} \\
{[\mathrm{mag}]}\end{array}$ & $\begin{array}{c}\mathrm{CN}_{2} \\
{[\mathrm{mag}]}\end{array}$ & $\begin{array}{c}\mathrm{Ca} 4227 \\
{[\AA]}\end{array}$ & $\begin{array}{c}\text { G4300 } \\
{[\AA]}\end{array}$ & $\begin{array}{c}\mathrm{H} \gamma_{\mathrm{A}} \\
{[\AA]}\end{array}$ & $\begin{array}{c}\mathrm{H} \gamma_{\mathrm{F}} \\
{[\AA]}\end{array}$ & $\begin{array}{c}\mathrm{Fe} 4384 \\
{[\AA]}\end{array}$ & $\begin{array}{c}\mathrm{Ca} 4455 \\
{[\AA]}\end{array}$ & $\begin{array}{c}\mathrm{Fe} 4531 \\
{[\AA]}\end{array}$ & $\begin{array}{c}\mathrm{C}_{2} 4668 \\
{[\AA]}\end{array}$ & $\begin{array}{l}\mathrm{H} \beta \\
{[\AA]}\end{array}$ & $\begin{array}{c}\text { Fe5015 } \\
{[\AA]}\end{array}$ \\
\hline \multirow[t]{2}{*}{$126-184$} & 3.82 & 3.03 & -0.060 & -0.029 & 0.42 & 2.44 & 1.80 & 2.77 & 1.06 & 0.26 & 1.43 & 0.75 & 3.63 & 2.29 \\
\hline & 0.53 & 0.47 & 0.017 & 0.018 & 0.17 & 0.30 & 0.29 & 0.17 & 0.50 & 0.21 & 0.31 & 0.44 & 0.14 & 0.37 \\
\hline \multirow[t]{2}{*}{$\dddot{13} 4-190$} & 0.58 & 0.91 & 0.010 & 0.040 & 0.64 & 4.53 & -2.57 & 0.34 & 2.86 & 0.49 & 2.38 & 1.73 & 1.76 & 2.85 \\
\hline & 0.52 & 0.47 & 0.017 & 0.018 & 0.17 & 0.28 & 0.31 & 0.19 & 0.50 & 0.21 & 0.31 & 0.45 & 0.16 & 0.38 \\
\hline \multirow[t]{2}{*}{$\dddot{15} \dot{5} 8-213$} & $0.2 \overline{7}$ & 1.10 & 0.040 & 0.068 & 0.62 & 4.06 & -2.53 & 0.18 & 2.91 & 0.63 & 2.40 & 1.97 & 1.72 & 3.23 \\
\hline & 0.44 & 0.38 & 0.015 & 0.016 & 0.09 & 0.16 & 0.19 & 0.12 & 0.37 & 0.12 & 0.19 & 0.28 & 0.10 & 0.27 \\
\hline \multirow[t]{2}{*}{$163-217$} & -0.40 & 0.68 & 0.160 & 0.207 & 1.03 & 5.06 & -5.26 & -1.02 & 4.52 & 1.25 & 3.37 & 4.06 & 1.72 & 4.79 \\
\hline & 0.43 & 0.37 & 0.014 & 0.015 & 0.07 & 0.12 & 0.16 & 0.09 & 0.33 & 0.09 & 0.15 & 0.21 & 0.07 & 0.22 \\
\hline \multirow[t]{2}{*}{$\dddot{2} 22-277$} & 6.98 & 4.60 & -0.120 & -0.076 & 0.72 & 1.87 & 4.06 & 3.57 & 1.86 & 0.78 & 2.74 & 1.39 & 4.44 & 4.10 \\
\hline & 0.67 & 0.62 & 0.022 & 0.025 & 0.31 & 0.57 & 0.51 & 0.31 & 0.85 & 0.40 & 0.59 & 0.89 & 0.31 & 0.72 \\
\hline \multirow[t]{2}{*}{$\dddot{22} 5-280$} & -0.88 & 0.64 & 0.090 & 0.129 & 0.78 & 4.57 & -3.71 & -0.22 & 3.82 & 0.99 & 2.84 & 2.95 & 1.81 & 4.25 \\
\hline & 0.43 & 0.37 & 0.014 & 0.015 & 0.07 & 0.12 & 0.16 & 0.09 & 0.34 & 0.09 & 0.16 & 0.22 & 0.07 & 0.23 \\
\hline \multirow[t]{2}{*}{$234-290$} & -0.10 & 0.85 & 0.000 & 0.030 & 0.75 & 4.37 & -3.21 & -0.18 & 2.49 & 0.52 & 2.56 & $1.7 \overline{7}$ & 1.70 & 3.14 \\
\hline & 0.51 & 0.45 & 0.016 & 0.018 & 0.15 & 0.26 & 0.30 & 0.18 & 0.48 & 0.20 & 0.30 & 0.44 & 0.16 & 0.38 \\
\hline \multirow[t]{2}{*}{$292-010$} & 4.47 & 3.39 & -0.070 & -0.028 & 0.29 & 2.45 & 1.79 & 2.55 & 0.82 & 0.14 & 0.66 & 1.23 & 3.12 & 1.67 \\
\hline & 0.64 & 0.58 & 0.021 & 0.023 & 0.29 & 0.51 & 0.50 & 0.30 & 0.82 & 0.39 & 0.60 & 0.88 & 0.32 & $\circledast 76$ \\
\hline \multirow{2}{*}{ 301-022 } & 3.60 & 2.17 & -0.080 & -0.056 & 0.89 & 3.02 & 0.85 & 1.91 & 1.21 & 0.43 & 1.73 & 0.72 & 2.92 & 2.26 \\
\hline & 0.78 & 0.76 & 0.024 & 0.027 & 0.35 & 0.63 & 0.61 & 0.38 & 0.94 & 0.44 & 0.65 & 0.95 & 0.33 & 0.74 \\
\hline \multirow{2}{*}{ 304-028 } & 1.94 & 2.05 & -0.050 & -0.019 & 0.32 & 2.29 & 1.13 & 1.87 & 0.57 & 0.23 & 0.68 & -0.18 & 2.50 & 0.59 \\
\hline & 0.69 & 0.64 & 0.021 & 0.024 & 0.30 & 0.53 & 0.51 & 0.32 & 0.82 & 0.39 & 0.59 & 0.86 & 0.30 & 0.72 \\
\hline \multirow{2}{*}{ 310-032 } & 4.07 & 2.98 & -0.110 & -0.072 & 0.44 & 1.77 & 1.34 & 1.85 & 0.62 & 0.01 & 1.26 & -0.22 & 2.54 & 1.45 \\
\hline & 0.68 & 0.64 & 0.021 & 0.024 & 0.31 & 0.55 & 0.52 & 0.32 & 0.84 & 0.40 & 0.59 & 0.86 & 0.30 & 0.70 \\
\hline \multirow{2}{*}{ 313-036 } & 0.51 & 0.96 & 0.010 & 0.046 & 0.75 & 4.20 & -2.75 & -0.01 & 2.53 & 0.60 & 2.14 & 2.09 & 1.49 & 3.00 \\
\hline & 0.56 & 0.51 & 0.017 & 0.019 & 0.20 & 0.34 & 0.37 & 0.23 & 0.57 & 0.25 & 0.38 & 0.56 & 0.21 & 0.48 \\
\hline \multirow{2}{*}{$\begin{array}{l}314-037 \\
\ldots\end{array}$} & 7.85 & 4.83 & -0.170 & -0.141 & 0.94 & 0.45 & 6.59 & 5.07 & -0.12 & 1.31 & 3.28 & 1.57 & 4.70 & 1.70 \\
\hline & 0.81 & 0.77 & 0.028 & 0.032 & 0.43 & 0.87 & 0.71 & 0.42 & 1.29 & 0.61 & 0.92 & 1.45 & 0.52 & 1.28 \\
\hline \multirow[t]{2}{*}{$321-046$} & 10.69 & 7.55 & -0.220 & -0.149 & 0.25 & -1.98 & 9.47 & 6.97 & 0.44 & 0.56 & 1.00 & 0.33 & 6.83 & 0.97 \\
\hline & 0.61 & 0.54 & 0.022 & 0.024 & 0.32 & 0.63 & 0.45 & 0.26 & 0.89 & 0.42 & 0.66 & 0.98 & 0.32 & 0.85 \\
\hline \multirow[t]{2}{*}{$\dddot{3} 22-049$} & 7.88 & 5.77 & -0.160 & -0.106 & 0.01 & -1.68 & 7.09 & 5.53 & -0.06 & 0.00 & 0.93 & 0.83 & 5.04 & 1.23 \\
\hline & 0.52 & 0.46 & 0.018 & 0.020 & 0.21 & 0.41 & 0.33 & 0.19 & 0.64 & 0.30 & 0.46 & 0.69 & 0.24 & 0.62 \\
\hline \multirow{2}{*}{$324-051$} & 6.75 & 4.83 & -0.120 & -0.069 & 0.29 & 0.84 & 4.89 & 4.42 & 0.74 & 0.36 & 2.34 & 2.11 & 4.67 & 3.24 \\
\hline & 0.46 & 0.40 & 0.016 & 0.017 & 0.13 & 0.24 & 0.23 & 0.13 & 0.45 & 0.18 & 0.27 & 0.42 & 0.14 & 0.38 \\
\hline \multirow[t]{2}{*}{$327-053$} & 5.85 & 4.40 & -0.080 & -0.030 & 0.05 & -1.03 & 5.56 & 4.58 & 0.37 & 0.52 & 0.72 & 1.04 & 3.76 & 1.78 \\
\hline & 0.45 & 0.39 & 0.015 & 0.016 & 0.12 & 0.24 & 0.21 & 0.12 & 0.44 & 0.17 & 0.28 & 0.41 & 0.14 & 0.38 \\
\hline \multirow{2}{*}{$\begin{array}{l}328-054 \\
\ldots\end{array}$} & 3.98 & 2.72 & -0.090 & -0.057 & 0.75 & 2.17 & 1.51 & 2.36 & 0.46 & 0.32 & 0.56 & -0.22 & 2.56 & 0.78 \\
\hline & 0.70 & $0.6 \overline{6}$ & 0.022 & 0.025 & 0.32 & 0.60 & 0.57 & 0.34 & 0.93 & 0.44 & 0.68 & 0.99 & 0.35 & 0.81 \\
\hline 337-068 & 2.66 & 2.29 & -0.060 & -0.030 & 0.60 & 3.73 & -0.02 & 1.79 & 2.22 & 0.58 & 2.10 & 1.60 & 3.21 & 2.65 \\
\hline & 0.43 & 0.37 & 0.015 & 0.015 & 0.07 & 0.13 & 0.16 & 0.09 & 0.34 & 0.09 & 0.16 & 0.22 & 0.07 & 0.22 \\
\hline $347-154$ & 4.38 & 3.12 & -0.080 & -0.036 & 0.29 & 1.24 & 2.83 & 2.89 & 0.52 & 0.03 & 1.01 & 0.51 & 2.85 & 1.01 \\
\hline & 0.51 & 0.45 & 0.017 & 0.018 & 0.17 & 0.31 & 0.29 & 0.18 & 0.53 & 0.22 & 0.34 & 0.50 & 0.17 & 0.43 \\
\hline $350-162$ & 3.50 & 2.69 & -0.050 & -0.013 & 0.35 & 2.55 & 1.11 & 2.12 & 1.29 & 0.21 & 1.20 & 0.89 & 2.78 & 1.59 \\
\hline & 0.59 & 0.54 & 0.019 & 0.021 & 0.25 & 0.43 & 0.43 & 0.26 & 0.70 & 0.33 & 0.50 & 0.74 & 0.27 & 0.63 \\
\hline $365-284$ & 2.83 & 2.47 & -0.060 & -0.025 & 0.32 & 2.53 & 1.33 & 2.35 & 1.12 & 0.33 & 1.53 & 0.71 & 2.70 & 2.05 \\
\hline & 0.52 & 0.46 & 0.017 & 0.018 & 0.17 & 0.30 & 0.30 & 0.18 & 0.53 & 0.22 & 0.34 & 0.50 & 0.17 & 0.43 \\
\hline $380-313$ & 9.60 & 6.46 & -0.190 & -0.123 & 0.41 & -0.17 & 8.20 & 6.32 & -0.08 & 0.37 & 1.84 & 0.61 & 6.05 & 2.74 \\
\hline & 0.50 & 0.44 & 0.017 & 0.019 & 0.19 & 0.38 & 0.31 & 0.17 & 0.61 & 0.27 & 0.41 & 0.63 & 0.22 & 0.57 \\
\hline & & & & & & & & & & & & & & \\
\hline
\end{tabular}


Table 4-Continued

\begin{tabular}{|c|c|c|c|c|c|c|c|c|c|c|c|c|c|c|}
\hline Name & $\begin{array}{c}\mathrm{H} \delta_{\mathrm{A}} \\
{[\AA]}\end{array}$ & $\begin{array}{l}\mathrm{H} \delta_{\mathrm{F}} \\
{[\AA]}\end{array}$ & $\begin{array}{c}\mathrm{CN}_{1} \\
{[\mathrm{mag}]}\end{array}$ & $\begin{array}{c}\mathrm{CN}_{2} \\
{[\mathrm{mag}]}\end{array}$ & $\begin{array}{c}\mathrm{Ca} 4227 \\
{[\AA]}\end{array}$ & $\begin{array}{c}\mathrm{G} 4300 \\
{[\AA]}\end{array}$ & $\begin{array}{c}\mathrm{H} \gamma_{\mathrm{A}} \\
{[\AA]}\end{array}$ & $\begin{array}{c}\mathrm{H} \gamma_{\mathrm{F}} \\
{[\AA]}\end{array}$ & $\begin{array}{c}\mathrm{Fe} 4384 \\
{[\AA]}\end{array}$ & $\begin{array}{c}\mathrm{Ca} 4455 \\
{[\AA]}\end{array}$ & $\begin{array}{c}\text { Fe4531 } \\
{[\AA]}\end{array}$ & $\begin{array}{c}\mathrm{C}_{2} 4668 \\
{[\AA]}\end{array}$ & $\begin{array}{l}\mathrm{H} \beta \\
{[\AA]}\end{array}$ & $\begin{array}{c}\text { Fe5015 } \\
{[\AA]}\end{array}$ \\
\hline $401-344$ & 4.51 & 3.55 & -0.090 & -0.054 & 0.01 & 1.49 & 2.69 & 2.75 & 0.21 & -0.01 & 0.40 & -0.26 & 2.82 & 0.59 \\
\hline & 0.49 & 0.44 & 0.017 & 0.018 & 0.17 & 0.31 & 0.29 & 0.18 & 0.53 & 0.23 & 0.35 & 0.53 & 0.19 & 0.47 \\
\hline N̈B16 & 3.52 & 2.77 & -0.070 & -0.035 & 0.50 & 2.71 & 1.30 & 2.38 & 1.45 & 0.37 & 1.66 & 1.22 & 3.32 & 2.31 \\
\hline & 0.43 & 0.37 & 0.015 & 0.015 & 0.07 & 0.14 & 0.16 & 0.09 & 0.35 & 0.10 & 0.17 & 0.25 & 0.08 & 0.25 \\
\hline NB67 & 3.29 & 2.71 & -0.070 & -0.033 & 0.48 & 2.73 & 1.20 & 2.26 & 1.44 & 0.29 & 1.76 & 1.09 & 3.29 & 2.38 \\
\hline & 0.45 & 0.39 & 0.015 & 0.016 & 0.11 & 0.20 & 0.21 & 0.12 & 0.41 & 0.15 & 0.23 & 0.34 & 0.12 & 0.32 \\
\hline N̈B68 & -0.15 & 0.94 & -0.040 & -0.017 & 0.94 & 5.23 & -3.69 & -0.43 & 3.50 & 0.65 & 2.50 & 2.45 & 2.30 & 3.09 \\
\hline & 0.45 & 0.39 & 0.015 & 0.016 & 0.10 & 0.18 & 0.21 & 0.13 & 0.38 & 0.13 & 0.21 & 0.30 & 0.10 & 0.28 \\
\hline NB74 & -2.90 & -0.58 & -0.020 & 0.001 & 1.53 & 6.44 & -6.92 & -2.24 & 5.29 & 0.67 & 2.47 & 2.12 & 1.22 & 2.95 \\
\hline & 0.84 & 0.78 & 0.024 & 0.027 & 0.33 & 0.58 & 0.73 & 0.46 & 0.90 & 0.45 & 0.66 & 1.00 & 0.38 & 0.86 \\
\hline NB81 & 0.58 & 1.20 & -0.040 & -0.016 & 0.75 & 5.25 & -3.15 & 0.15 & 3.79 & 0.95 & 2.76 & 3.85 & 2.83 & 4.05 \\
\hline & 0.46 & 0.41 & 0.015 & 0.016 & 0.12 & 0.20 & 0.24 & 0.15 & 0.41 & 0.15 & 0.24 & 0.35 & 0.12 & 0.33 \\
\hline NB83 & 2.61 & 2.27 & -0.060 & -0.023 & 0.66 & 3.56 & 0.22 & 1.96 & 1.97 & 0.49 & 2.09 & 1.62 & 3.21 & 2.63 \\
\hline & 0.46 & 0.41 & 0.015 & 0.016 & 0.12 & 0.22 & 0.24 & 0.14 & 0.43 & 0.17 & 0.26 & 0.38 & 0.13 & 0.35 \\
\hline NB87 & -3.72 & -0.56 & 0.010 & 0.048 & 1.76 & 5.96 & -7.38 & -2.19 & 6.22 & 1.21 & 3.24 & 4.52 & 1.59 & 4.29 \\
\hline & 0.50 & 0.43 & 0.016 & 0.017 & 0.12 & 0.22 & 0.28 & 0.17 & 0.42 & 0.16 & 0.25 & 0.36 & 0.13 & E. 33 \\
\hline NB89 & 0.70 & 1.31 & 0.050 & 0.094 & 0.69 & 4.04 & -1.61 & 0.84 & 2.85 & 0.88 & 2.65 & 2.11 & 2.02 & 3.28 \\
\hline & 0.50 & 0.44 & 0.016 & 0.017 & 0.13 & 0.22 & 0.23 & 0.14 & 0.41 & 0.14 & 0.22 & 0.30 & 0.09 & 0.26 \\
\hline NB91 & -0.38 & 0.84 & -0.040 & -0.016 & 0.95 & 5.25 & -3.76 & -0.53 & 3.59 & 0.70 & 2.43 & 2.42 & 2.17 & 3.32 \\
\hline$\ldots$ & 0.47 & 0.41 & 0.015 & 0.016 & 0.11 & 0.20 & 0.23 & 0.15 & 0.40 & 0.14 & 0.23 & 0.33 & 0.12 & 0.30 \\
\hline
\end{tabular}


Table 4A. Lick/IDS indices and uncertainties for M31 globular clusters

\begin{tabular}{|c|c|c|c|c|c|c|c|c|c|c|}
\hline Name & $\begin{array}{c}\mathrm{Mg}_{1} \\
{[\mathrm{mag}]}\end{array}$ & $\begin{array}{c}\mathrm{Mg}_{2} \\
{[\mathrm{mag}]}\end{array}$ & $\begin{array}{c}\operatorname{Mg} b \\
{[\AA]}\end{array}$ & $\begin{array}{c}\text { Fe } 5270 \\
{[\AA]}\end{array}$ & $\begin{array}{c}\text { Fe5335 } \\
{[\AA]}\end{array}$ & $\begin{array}{c}\text { Fe } 5406 \\
{[\AA]}\end{array}$ & $\begin{array}{c}\text { Fe5709 } \\
{[\AA]}\end{array}$ & $\begin{array}{c}\text { Fe5782 } \\
{[\AA]}\end{array}$ & $\begin{array}{c}\mathrm{Na} \mathrm{D} \\
{[\AA]}\end{array}$ & $\begin{array}{l}\mathrm{TiO}_{1} \\
{[\mathrm{mag}]}\end{array}$ \\
\hline \multirow[t]{2}{*}{$126-184$} & 0.014 & 0.066 & 1.28 & 1.02 & 0.81 & 0.47 & 0.27 & 0.16 & 1.15 & 0.010 \\
\hline & 0.015 & 0.014 & 0.24 & 0.17 & 0.20 & 0.14 & 0.11 & 0.11 & 0.52 & 0.010 \\
\hline \multirow[t]{2}{*}{$\dddot{13} 4-190$} & 0.050 & 0.129 & 2.22 & 1.79 & 1.59 & 0.99 & 0.61 & 0.41 & 1.75 & 0.022 \\
\hline & 0.015 & 0.014 & 0.24 & 0.17 & 0.20 & 0.15 & 0.11 & 0.11 & 0.52 & 0.010 \\
\hline \multirow[t]{2}{*}{$158-213$} & 0.074 & 0.150 & 2.27 & 1.86 & 1.70 & 1.06 & 0.62 & 0.47 & 2.48 & 0.020 \\
\hline & 0.015 & 0.013 & 0.21 & 0.10 & 0.13 & 0.09 & 0.07 & 0.07 & 0.51 & 0.010 \\
\hline \multirow[t]{2}{*}{$163-217$} & 0.098 & 0.242 & 4.01 & 2.60 & 2.44 & 1.57 & 0.83 & 0.76 & 4.30 & 0.038 \\
\hline & 0.015 & 0.013 & 0.19 & 0.07 & 0.09 & 0.06 & 0.04 & 0.05 & 0.50 & 0.010 \\
\hline \multirow[t]{2}{*}{$222-277$} & 0.050 & 0.121 & 1.30 & 1.87 & 1.17 & 0.97 & 0.61 & 0.37 & 3.25 & 0.029 \\
\hline & 0.016 & 0.015 & 0.39 & 0.37 & 0.43 & 0.31 & 0.24 & 0.24 & 0.58 & 0.012 \\
\hline \multirow[t]{2}{*}{$\dddot{22} 25-280$} & 0.089 & 0.207 & 3.21 & 2.31 & 2.03 & 1.31 & 0.79 & 0.65 & 3.10 & 0.027 \\
\hline & 0.015 & 0.013 & 0.19 & 0.07 & 0.09 & 0.06 & 0.05 & 0.05 & 0.50 & 0.010 \\
\hline \multirow[t]{2}{*}{$234-290$} & 0.055 & 0.133 & 2.37 & 2.03 & 1.50 & 0.99 & 0.51 & 0.46 & 2.15 & 0.020 \\
\hline & 0.015 & 0.014 & 0.24 & 0.18 & 0.21 & 0.15 & 0.12 & 0.11 & 0.52 & 0.010 \\
\hline \multirow[t]{2}{*}{ 292-010 } & 0.038 & 0.073 & 0.97 & 0.95 & 1.14 & 0.15 & 0.33 & 0.19 & 1.32 & 0.006 \\
\hline & 0.016 & 0.016 & 0.40 & 0.39 & 0.45 & 0.34 & 0.26 & 0.25 & 0.60 & 0.013 \\
\hline \multirow[t]{2}{*}{$301-022$} & 0.022 & 0.076 & 1.70 & 1.43 & 1.18 & 0.33 & 0.56 & 0.36 & 1.44 & 0.008 \\
\hline & 0.016 & 0.015 & 0.38 & 0.37 & 0.42 & 0.31 & 0.24 & 0.23 & 0.58 & 0.012 \\
\hline \multirow[t]{2}{*}{$\dddot{304}-028$} & 0.041 & 0.070 & 1.23 & 1.40 & 0.92 & 0.85 & 0.39 & 0.26 & 1.72 & 0.011 \\
\hline & 0.016 & 0.015 & 0.37 & 0.36 & 0.42 & 0.30 & 0.24 & 0.23 & 0.58 & 0.012 \\
\hline \multirow[t]{2}{*}{$\dddot{310-032}$} & 0.035 & 0.055 & 0.92 & 1.26 & 0.84 & 0.44 & 0.25 & 0.02 & 1.57 & 0.017 \\
\hline & 0.016 & 0.015 & 0.36 & 0.35 & 0.40 & 0.29 & 0.23 & 0.22 & 0.57 & 0.012 \\
\hline \multirow[t]{2}{*}{ 313-036 } & 0.060 & 0.140 & 2.17 & 1.69 & 1.45 & 0.97 & 0.66 & 0.61 & 2.80 & 0.024 \\
\hline & 0.015 & 0.014 & 0.28 & 0.23 & 0.26 & 0.19 & 0.15 & 0.14 & 0.53 & 0.011 \\
\hline \multirow[t]{2}{*}{ 314-037 } & 0.055 & 0.122 & 2.85 & 1.56 & 1.89 & 0.82 & 0.80 & 0.31 & 2.73 & -0.004 \\
\hline & 0.020 & 0.020 & 0.61 & 0.69 & 0.79 & 0.59 & 0.47 & 0.47 & 0.77 & 0.017 \\
\hline \multirow[t]{2}{*}{$321-046$} & 0.028 & 0.052 & 0.94 & 0.80 & 0.73 & 0.20 & 0.45 & 0.35 & 1.37 & 0.007 \\
\hline & 0.017 & 0.016 & 0.43 & 0.45 & 0.52 & 0.38 & 0.30 & 0.29 & 0.62 & 0.013 \\
\hline \multirow[t]{2}{*}{$\dddot{3} 22-049$} & 0.038 & 0.048 & 0.35 & 0.63 & 0.67 & 0.45 & 0.46 & 0.28 & 1.68 & 0.014 \\
\hline & 0.016 & 0.015 & 0.34 & 0.33 & 0.38 & 0.28 & 0.23 & 0.22 & 0.58 & 0.012 \\
\hline \multirow[t]{2}{*}{ 324-051 } & 0.045 & 0.085 & 1.57 & 1.66 & 1.46 & 0.71 & 0.55 & 0.31 & 0.71 & 0.019 \\
\hline & 0.015 & 0.014 & 0.24 & 0.19 & 0.22 & 0.16 & 0.13 & 0.13 & 0.53 & 0.011 \\
\hline \multirow[t]{2}{*}{$327-053$} & 0.050 & 0.077 & 0.59 & 0.83 & 1.10 & 0.72 & 0.42 & 0.60 & 2.67 & 0.028 \\
\hline & 0.015 & 0.014 & 0.25 & 0.19 & 0.22 & 0.16 & 0.13 & 0.12 & 0.52 & 0.011 \\
\hline \multirow[t]{2}{*}{ 328-054 } & 0.033 & 0.068 & 0.19 & 0.89 & 0.49 & 0.40 & 0.04 & 0.44 & 1.88 & 0.002 \\
\hline & 0.017 & 0.016 & 0.42 & 0.41 & 0.48 & 0.35 & 0.27 & 0.25 & 0.59 & 0.013 \\
\hline \multirow{2}{*}{$\ddot{3} 37-068$} & 0.013 & 0.084 & 1.86 & 1.48 & 1.11 & 0.64 & 0.44 & 0.26 & 1.21 & 0.006 \\
\hline & 0.015 & 0.013 & 0.19 & 0.07 & 0.09 & 0.06 & 0.05 & 0.05 & 0.50 & 0.010 \\
\hline \multirow[t]{2}{*}{$347-154$} & 0.020 & 0.044 & 0.76 & 0.51 & 0.49 & 0.37 & 0.12 & 0.11 & 1.15 & 0.002 \\
\hline & 0.015 & 0.014 & 0.26 & 0.21 & 0.24 & 0.17 & 0.14 & 0.13 & 0.53 & 0.011 \\
\hline 350-162 & 0.034 & 0.075 & 1.13 & 0.98 & 0.81 & 0.33 & 0.39 & 0.19 & 1.00 & 0.006 \\
\hline & 0.016 & 0.015 & 0.34 & 0.32 & 0.37 & 0.27 & 0.21 & 0.20 & 0.56 & 0.012 \\
\hline $365-284$ & 0.040 & 0.080 & 1.37 & 1.33 & 1.00 & 0.50 & 0.43 & 0.31 & 2.54 & 0.014 \\
\hline & 0.015 & 0.014 & 0.26 & 0.20 & 0.24 & 0.18 & 0.14 & 0.13 & 0.53 & 0.011 \\
\hline $380-313$ & 0.038 & 0.080 & 1.19 & 1.86 & 1.36 & 0.64 & 0.50 & 0.42 & 1.96 & 0.026 \\
\hline & 0.016 & 0.014 & 0.32 & 0.29 & 0.34 & 0.26 & 0.21 & 0.20 & 0.56 & 0.012 \\
\hline
\end{tabular}


Table 4A-Continued

\begin{tabular}{|c|c|c|c|c|c|c|c|c|c|c|}
\hline$\overline{\text { Name }}$ & $\begin{array}{l}\mathrm{Mg}_{1} \\
{[\mathrm{mag}]}\end{array}$ & $\begin{array}{l}\mathrm{Mg}_{2} \\
{[\mathrm{mag}]}\end{array}$ & $\begin{array}{c}\overline{\operatorname{Mg} b} \\
{[\AA]}\end{array}$ & $\begin{array}{c}\text { Fe5270 } \\
{[\AA]}\end{array}$ & $\begin{array}{c}\text { Fe5335 } \\
{[\AA]}\end{array}$ & $\begin{array}{c}\text { Fe5406 } \\
{[\AA]}\end{array}$ & $\begin{array}{c}\text { Fe5709 } \\
{[\AA]}\end{array}$ & $\begin{array}{c}\text { Fe5782 } \\
{[\AA]}\end{array}$ & $\begin{array}{c}\mathrm{Na} \mathrm{D} \\
{[\AA]}\end{array}$ & $\begin{array}{l}\overline{\mathrm{TiO}_{1}} \\
{[\mathrm{mag}]}\end{array}$ \\
\hline & 0.015 & 0.014 & 0.27 & 0.23 & 0.27 & 0.20 & 0.16 & 0.15 & 0.54 & 0.011 \\
\hline \multirow[t]{2}{*}{ N̈B16 } & 0.023 & 0.086 & 1.61 & 1.18 & 0.97 & 0.50 & 0.39 & 0.20 & 1.16 & 0.006 \\
\hline & 0.015 & 0.013 & 0.20 & 0.09 & 0.11 & 0.08 & 0.06 & 0.07 & 0.51 & 0.010 \\
\hline \multirow{2}{*}{ NB67 } & 0.023 & 0.081 & 1.65 & 1.12 & 0.79 & 0.47 & 0.32 & 0.23 & 1.14 & 0.003 \\
\hline & 0.015 & 0.013 & 0.22 & 0.14 & 0.17 & 0.12 & 0.09 & 0.09 & 0.51 & 0.010 \\
\hline \multirow[t]{2}{*}{ NB68 } & 0.033 & 0.145 & 3.22 & 1.98 & 1.59 & 0.90 & 0.59 & 0.40 & 1.51 & 0.005 \\
\hline & 0.015 & 0.013 & 0.21 & 0.12 & 0.14 & 0.10 & 0.08 & 0.08 & 0.51 & 0.010 \\
\hline \multirow[t]{2}{*}{ NB74 } & 0.080 & 0.239 & 5.17 & 1.82 & 1.83 & 0.97 & 0.72 & 0.34 & 3.10 & -0.003 \\
\hline & 0.017 & 0.016 & 0.42 & 0.45 & 0.52 & 0.39 & 0.31 & 0.30 & 0.62 & 0.013 \\
\hline \multirow[t]{2}{*}{ NB81 } & 0.041 & 0.142 & 2.72 & 2.19 & 1.75 & 0.90 & 0.78 & 0.48 & 1.51 & 0.002 \\
\hline & 0.015 & 0.013 & 0.22 & 0.14 & 0.17 & 0.12 & 0.10 & 0.10 & 0.51 & 0.010 \\
\hline \multirow[t]{2}{*}{ NB83 } & 0.023 & 0.087 & 1.79 & 1.44 & 1.09 & 0.42 & 0.38 & 0.26 & 1.18 & 0.004 \\
\hline & 0.015 & 0.014 & 0.23 & 0.16 & 0.19 & 0.14 & 0.11 & 0.11 & 0.52 & 0.010 \\
\hline \multirow[t]{2}{*}{ NB87 } & 0.089 & 0.277 & 5.19 & 3.25 & 2.85 & 1.88 & 0.98 & 0.75 & 2.99 & 0.006 \\
\hline & 0.015 & 0.013 & 0.22 & 0.14 & 0.17 & 0.12 & 0.09 & 0.09 & 0.51 & 0.010 \\
\hline \multirow[t]{2}{*}{ NB89 } & 0.054 & 0.143 & 2.43 & 1.91 & 1.63 & 1.02 & 0.59 & 0.50 & 3.08 & 0.019 \\
\hline & 0.015 & 0.013 & 0.20 & 0.09 & 0.11 & 0.07 & 0.06 & 0.06 & 0.50 & 0.010 \\
\hline \multirow{2}{*}{$\begin{array}{l}\text { NB91 } \\
\ldots\end{array}$} & 0.034 & 0.145 & 3.19 & 1.96 & 1.76 & 0.98 & 0.65 & 0.37 & 1.79 & 0.004 \\
\hline & 0.015 & 0.013 & 0.21 & 0.13 & 0.16 & 0.11 & 0.09 & 0.09 & 0.51 & 0.010 \\
\hline
\end{tabular}


Table 5. Linear fit coefficients, rms of linear fit, mean difference and its standard deviation between the CBR98 Galactic cluster data and Trager et al. (1998) Galactic clusters. Lick indices for $\mathrm{H} \delta$ and $\mathrm{H} \gamma$ are taken from Kuntschner et al. (2003). N signifies number of clusters with common indices. Indices measured using the Trager et al. (1998) and Worthey \& Ottaviani (1997) Lick index definitions.

\begin{tabular}{lllcccc}
\hline \hline Index & \multicolumn{1}{c}{$a$} & $b$ & rms & Lick-CBR98 & $\sigma_{\text {rms }}$ & $\mathrm{N}$ \\
& & & & & & \\
\hline $\mathrm{H} \delta_{\mathrm{A}}$ & $\ldots$ & $\ldots$ & $\ldots$ & $\ldots$ & $\ldots$ & $\ldots$ \\
$\mathrm{H} \delta_{\mathrm{F}}$ & 0.6108 & -1.3760 & $\ldots$ & 0.2210 & 1.1430 & $2^{a}$ \\
$\mathrm{CN}_{1}$ & 0.0725 & -1.0540 & $\ldots$ & 0.0210 & 0.0537 & $2^{a}$ \\
$\mathrm{CN}_{2}$ & 0.0052 & 0.8462 & $\ldots$ & 0.0020 & 0.0057 & $2^{a}$ \\
$\mathrm{Ca} 4227$ & 0.2373 & 0.4451 & 0.0631 & 0.1067 & 0.3919 & $4^{b}$ \\
$\mathrm{G} 4300$ & 0.2390 & 1.0550 & 0.4266 & -0.4015 & 0.5039 & $4^{b}$ \\
$\mathrm{H} \gamma_{\mathrm{A}}$ & -1.1790 & 2.4030 & 0.1657 & 0.5383 & 2.3030 & 3 \\
$\mathrm{H} \gamma_{\mathrm{F}}$ & -0.1537 & 0.9790 & 0.7687 & 0.1710 & 0.8882 & 4 \\
$\mathrm{Fe} 4383$ & 0.4878 & 0.5728 & 0.3304 & 0.6123 & 1.0930 & $4^{b}$ \\
$\mathrm{Ca} 4455$ & -0.1364 & 0.5420 & 0.0373 & 0.5220 & 0.2441 & 5 \\
$\mathrm{Fe} 4531$ & -0.3698 & 1.0900 & 0.0702 & 0.1987 & 0.1172 & $4^{b}$ \\
$\mathrm{C}_{2} 4668$ & 0.2719 & 0.7662 & 0.4248 & 0.0274 & 0.5502 & $5^{b}$ \\
$\mathrm{H} \beta$ & 0.2606 & 0.8592 & 0.1256 & 0.0077 & 0.1570 & 6 \\
$\mathrm{Fe} 5015$ & -0.3993 & 1.0290 & 0.2905 & 0.3152 & 0.3202 & 6 \\
$\mathrm{Mg} 1$ & 0.0002 & 1.1940 & 0.0171 & -0.0068 & 0.0192 & 6 \\
$\mathrm{Mg} 2$ & 0.0091 & 0.9802 & 0.0010 & -0.0070 & 0.0017 & 6 \\
$\mathrm{Mg} b$ & -0.0859 & 1.0300 & 0.1239 & 0.0275 & 0.1392 & 6 \\
$\mathrm{Fe} 5270$ & 0.0543 & 0.9417 & 0.2217 & 0.0348 & 0.2461 & 6 \\
$\mathrm{Fe} 5335$ & 0.3537 & 0.8841 & 0.2172 & -0.2305 & 0.2492 & 6 \\
$\mathrm{Fe} 5406$ & -0.1683 & 1.0310 & 0.0463 & 0.1390 & 0.0526 & 6 \\
$\mathrm{Fe} 5709$ & 0.1194 & 0.7490 & 0.1511 & 0.0106 & 0.1934 & $5^{b}$ \\
$\mathrm{Fe} 5782$ & 0.0567 & 0.8221 & 0.1144 & 0.0180 & 0.1345 & 6 \\
$\mathrm{NaD}_{2}$ & 0.1374 & 0.8028 & 0.1840 & 0.2898 & 0.2990 & 6 \\
$\mathrm{TiO} 1$ & 0.0078 & 0.5477 & 0.0068 & 0.0005 & 0.0112 & 6 \\
$\mathrm{TiO} 2$ & -0.0102 & 1.3910 & 0.0154 & -0.0052 & 0.0199 & 4 \\
\hline & & & & & & \\
\hline
\end{tabular}

${ }^{\mathrm{a} N o}$ rms calculated for $\mathrm{N}<3$.

${ }^{\mathrm{b}}$ Index measurement for NGC 6171 excluded from fit. 
Table 6. Linear fit coefficients, rms of linear fit, mean difference and its standard deviation between the three clusters in common between the LRIS M31 globular cluster data and Trager et al. (1998). Lick indices for $\mathrm{H} \delta$ and $\mathrm{H} \gamma$ are taken from Kuntschner et al. (2003). Lick index definitions are given in Trager et al. (1998) and Worthey \& Ottaviani (1997)

\begin{tabular}{lllcccc}
\hline \hline Index & \multicolumn{1}{c}{$a$} & \multicolumn{1}{c}{$b$} & $\mathrm{rms}$ & Lick-M31 & $\sigma_{\text {rms }}$ & $\mathrm{N}$ \\
& & & & & & \\
\hline $\mathrm{H} \delta_{\mathrm{A}}$ & -1.5190 & -0.4680 & $\ldots$ & -1.2330 & 1.0690 & $2^{a}$ \\
$\mathrm{H} \delta_{\mathrm{F}}$ & 0.6042 & 0.2634 & 0.0143 & -0.0370 & 0.7143 & 3 \\
$\mathrm{CN}_{1}$ & 0.0308 & 1.0480 & 0.0048 & -0.0337 & 0.0065 & 3 \\
$\mathrm{CN}_{2}$ & 0.0334 & 0.9869 & 0.0063 & -0.0320 & 0.0078 & 3 \\
$\mathrm{Ca} 4227$ & 0.4951 & 0.4415 & 0.1514 & -0.0967 & 0.2211 & 3 \\
$\mathrm{G} 4300$ & -0.1284 & 1.0120 & 0.1967 & 0.0733 & 0.2410 & 3 \\
$\mathrm{H} \gamma_{\mathrm{A}}$ & 5.3500 & 1.9070 & $\ldots$ & -0.6725 & 0.5197 & $2^{a}$ \\
$\mathrm{H} \gamma_{\mathrm{F}}$ & -0.2218 & 0.6410 & 0.1045 & 0.1500 & 0.3576 & 3 \\
$\mathrm{Fe} 4383$ & 4.5760 & -0.2190 & 0.6566 & 0.0233 & 0.9420 & 3 \\
$\mathrm{Ca} 4455$ & -0.3685 & 1.0790 & 0.0962 & 0.2720 & 0.1196 & 3 \\
$\mathrm{Fe} 4531$ & 1.0450 & 0.6931 & 0.0273 & -0.2370 & 0.2176 & 3 \\
$\mathrm{C}_{2} 4668$ & 1.8400 & 0.4738 & 0.2666 & -0.5613 & 1.1520 & 3 \\
$\mathrm{H} \beta$ & 1.4620 & 0.1640 & 0.0273 & 0.0150 & 0.2247 & 3 \\
$\mathrm{Fe} 5015$ & 0.9523 & 0.7859 & 0.2682 & -0.0973 & 0.3823 & 3 \\
$\mathrm{Mg} 1$ & 0.0646 & 0.4040 & 0.0066 & -0.0317 & 0.0156 & 3 \\
$\mathrm{Mg} 2$ & 0.0236 & 0.9728 & 0.0031 & -0.0187 & 0.0040 & 3 \\
$\mathrm{Mg} b$ & -0.5430 & 1.2040 & 0.0946 & -0.0840 & 0.1863 & 3 \\
$\mathrm{Fe} 5270$ & -0.0404 & 0.9489 & 0.1330 & 0.1640 & 0.1639 & 3 \\
$\mathrm{Fe} 5335$ & 0.4890 & 0.8229 & 0.1629 & -0.1513 & 0.2104 & 3 \\
$\mathrm{Fe} 5406$ & -0.5510 & 1.6110 & 0.1155 & -0.1560 & 0.1633 & 3 \\
$\mathrm{Fe} 5709$ & 0.4219 & 0.3616 & 0.0688 & 0.1527 & 0.1622 & 3 \\
$\mathrm{Fe} 5782$ & 0.4351 & 0.2569 & 0.0846 & 0.1173 & 0.3092 & 3 \\
$\mathrm{NaD}$ & -0.5590 & 1.1620 & 0.1179 & 0.0210 & 0.1932 & 3 \\
$\mathrm{TiO}{ }_{1}$ & 0.0186 & 0.4208 & 0.0032 & -0.0053 & 0.0119 & 3 \\
$\mathrm{TiO}{ }_{2}$ & $\ldots$ & $\ldots$ & $\ldots$ & $\ldots$ & $\ldots$ & $\ldots$ \\
\hline & & & & & &
\end{tabular}

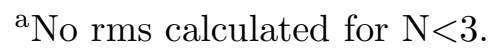


Table 7. Results of cross-correlation between young M31 clusters and the LMC templates of Beasley et al. 2002. LMC \#1..\#3 denote the first, second and third best template matches for the M31 cluster in question.

\begin{tabular}{|c|c|c|c|c|}
\hline ID & LMC \#1 & LMC \#2 & LMC \#3 & Age Range (Gyr) \\
\hline $327-053$ & SL106 (I) & NGC 2002(I) & NGC 1863(I) & $0.01-0.03$ \\
\hline $322-049$ & NGC 2031 (III) & NGC 1735 (II) & NGC 1815 (I) & $0.01-0.20$ \\
\hline $380-313$ & NGC 1735 (II) & NGC 2031 (III) & NGC 1755 (II) & $0.01-0.20$ \\
\hline $321-046$ & NGC 2031 (III) & NGC 1735 (II) & NGC 1774 (II) & $0.01-0.20$ \\
\hline $324-051$ & NGC 1940 (II) & NGC 2041 (II) & NGC 2127( III) & $0.01-0.20$ \\
\hline $314-037$ & NGC 2127 (III) & NGC 2041 (II) & NGC 1878 (IVA) & $0.03-0.40$ \\
\hline $222-277$ & NGC 1878 (IVA) & NGC2127 (III) & NGC $1806(\mathrm{~V})$ & $0.07-2.00$ \\
\hline $292-010$ & NGC $1852(\mathrm{~V})$ & NGC $1751(\mathrm{~V})$ & NGC 1718 (VI) & $0.80-2.0$ \\
\hline
\end{tabular}

Note. - Roman numerals in parenthesis denote SWB-type, originally defined by Searle, Wilkinson \& Bagnuolo (1980). Age-SWB calibration from Bica et al. (1992) 
Table 8. Best matches to the BC03 stellar population models using a $\chi^{2}$ minimization of our measured Lick indices for the young M31 clusters. N refers to the number of Lick indices utilised in the fitting procedure. Re-measured velocities for the clusters using new templates are also given.

\begin{tabular}{|c|c|c|c|c|c|c|}
\hline ID & {$[\mathrm{Fe} / \mathrm{H}]$} & Age(Gyr) & $\mathrm{N}$ & $M / L_{V}$ & $\operatorname{Mass}\left(\mathrm{M}_{\odot}\right)$ & Velocity $\left(\mathrm{kms}^{-1}\right)$ \\
\hline $327-053$ & $0.300 \pm 0.750$ & $0.080 \pm 0.929$ & 18 & 0.092 & $2.1 \times 10^{4}$ & $-542 \pm 56$ \\
\hline $322-049$ & $-0.200 \pm 0.200$ & $0.102 \pm 0.470$ & 24 & 0.104 & $7.0 \times 10^{3}$ & $-576 \pm 21$ \\
\hline $380-313$ & $0.150 \pm 0.100$ & $0.454 \pm 0.133$ & 24 & 0.250 & $4.1 \times 10^{4}$ & $-115 \pm 24$ \\
\hline $321-046$ & $0.000 \pm 0.600$ & $0.286 \pm 0.280$ & 24 & 0.178 & $1.4 \times 10^{4}$ & $-513 \pm 32$ \\
\hline $324-051$ & $-0.050 \pm 0.400$ & $0.839 \pm 0.167$ & 23 & 0.378 & $6.5 \times 10^{4}$ & $-266 \pm 38$ \\
\hline $314-037$ & $0.350 \pm 0.250$ & $0.509 \pm 0.590$ & 22 & 0.268 & $2.3 \times 10^{4}$ & $-484 \pm 54$ \\
\hline $222-277$ & $0.300 \pm 0.600$ & $0.719 \pm 0.716$ & 22 & 0.346 & $4.5 \times 10^{4}$ & $-320 \pm 55$ \\
\hline $292-010$ & $-1.350 \pm 0.550$ & $2.748 \pm 1.151$ & 22 & 1.110 & $1.7 \times 10^{5}$ & $-392 \pm 56$ \\
\hline
\end{tabular}

